\title{
Multidendritic sensory neurons in the adult Drosophila abdomen: origins, dendritic morphology, and segment- and age-dependent programmed cell death
}

\author{
Kohei Shimono $^{\dagger 1}$, Azusa Fujimoto ${ }^{\dagger 1}$, Taiichi Tsuyama ${ }^{1}$, Misato Yamamoto- \\ Kochi $^{1}$, Motohiko Sato ${ }^{1,2}$, Yukako Hattori ${ }^{1}$, Kaoru Sugimura ${ }^{1,3}$, Tadao Usui ${ }^{1}$, \\ Ken-ichi Kimura*4 and Tadashi Uemura*1
}

\begin{abstract}
Address: ${ }^{1}$ Laboratory of Cell Recognition and Pattern Formation, Graduate School of Biostudies, South Campus Research Building (Building G) Kyoto University, Yoshida Konoe-cho, Sakyo-ku, Kyoto 606-8507, Japan, ${ }^{2}$ Laboratory of Neural Development, Graduate School of Biostudies, Kyoto University, Kyoto 606-8501, Japan, ${ }^{3}$ Laboratory of Cell Function Dynamics, Advanced Technology Development Group, Brain Science Institute, RIKEN, Wako 351-0198, Japan and ${ }^{4}$ Hokkaido University of Education, Sapporo Campus, Sapporo 002-8502, Japan

Email: Kohei Shimono - kshimono@lif.kyoto-u.ac.jp; Azusa Fujimoto - afujimoto@lif.kyoto-u.ac.jp; Taiichi Tsuyama - t-tsuyama@lif.kyotou.ac.jp; Misato Yamamoto-Kochi - myamamoto@lif.kyoto-u.ac.jp; Motohiko Sato - msato@lif.kyoto-u.ac.jp;

Yukako Hattori - yhattori@lif.kyoto-u.ac.jp; Kaoru Sugimura - ksugimura@brain.riken.jp; Tadao Usui - tusui@lif.kyoto-u.ac.jp; Ken-

ichi Kimura* - kimurakn@sap.hokkyodai.ac.jp; Tadashi Uemura* - tauemura@lif.kyoto-u.ac.jp

* Corresponding authors †Equal contributors
\end{abstract}

Published: 2 October 2009

Neural Development 2009, 4:37 doi:10.1186/1749-8104-4-37
Received: 22 April 2009

Accepted: 2 October 2009

This article is available from: http://www.neuraldevelopment.com/content/4/I/37

(c) 2009 Shimono et al; licensee BioMed Central Ltd.

This is an Open Access article distributed under the terms of the Creative Commons Attribution License (http://creativecommons.org/licenses/by/2.0), which permits unrestricted use, distribution, and reproduction in any medium, provided the original work is properly cited.

\begin{abstract}
Background: For the establishment of functional neural circuits that support a wide range of animal behaviors, initial circuits formed in early development have to be reorganized. One way to achieve this is local remodeling of the circuitry hardwiring. To genetically investigate the underlying mechanisms of this remodeling, one model system employs a major group of Drosophila multidendritic sensory neurons - the dendritic arborization (da) neurons - which exhibit dramatic dendritic pruning and subsequent growth during metamorphosis. The 15 da neurons are identified in each larval abdominal hemisegment and are classified into four categories - classes I to IV - in order of increasing size of their receptive fields and/or arbor complexity at the mature larval stage. Our knowledge regarding the anatomy and developmental basis of adult da neurons is still fragmentary.

Results: We identified multidendritic neurons in the adult Drosophila abdomen, visualized the dendritic arbors of the individual neurons, and traced the origins of those cells back to the larval stage. There were six da neurons in abdominal hemisegment 3 or $4(\mathrm{~A} 3 / 4)$ of the pharate adult and the adult just after eclosion, five of which were persistent larval da neurons. We quantitatively analyzed dendritic arbors of three of the six adult neurons and examined expression in the pharate adult of key transcription factors that result in the larval class-selective dendritic morphologies. The 'baseline design' of A3/4 in the adult was further modified in a segment-dependent and age-dependent manner. One of our notable findings is that a larval class I neuron, ddaE, completed dendritic remodeling in A2 to A4 and then underwent caspasedependent cell death within I week after eclosion, while homologous neurons in A5 and in more posterior segments degenerated at pupal stages. Another finding is that the dendritic arbor of a class IV neuron, v'ada, was immediately reshaped during post-eclosion growth. It exhibited prominent radial-to-lattice transformation in I-day-old adults, and the resultant lattice-shaped arbor persisted throughout adult life.
\end{abstract}

Conclusion: Our study provides the basis on which we can investigate the genetic programs controlling dendritic remodeling and programmed cell death of adult neurons, and the life-long maintenance of dendritic arbors. 


\section{Background}

The development of the nervous system is not complete at the end of embryogenesis; instead, it involves a series of progressive events. During the postembryonic phase, the nervous system is reorganized at multiple structural levels to strengthen, elaborate, and/or modify already acquired functions, and even add novel ones. This reorganization of initial neural circuits formed during early development is critical to support a wide range of animal behaviors under a variety of environmental contexts [1,2]. One cellular mechanism of this reorganization is the disposal of a subset of early-born neurons and replacement of those cells with later-born ones. Another is 'recycling,' which is accomplished by pruning of axons or dendrites without loss of parental neurons and concomitant or subsequent growth of these processes in spatially distinct patterns [35].

Both these cellular mechanisms are observed dramatically in the neural and motor systems of insects that undergo complete metamorphosis (holometabolous insects), which is necessary for the generation of stage-specific behaviors. Nerve and muscle cells that are required for larval behaviors, such as crawling and feeding, must either be replaced or re-specified to allow adult walking, flight, mating, and egg-laying $[2,6]$. This conversion from larva to adult behaviors is manifested, at least in part, by the remodeling of dendritic arbors. For example, in the early pupae of Manduca, an identified motoneuron forms additional dendritic branches, accepting new excitatory synapses from stretch receptors [7]. Therefore, remodeling of stage-specific dendritic patterns is an essential process to realize the proper function of the nervous system.

To genetically investigate underlying mechanisms of axon and dendrite remodeling, researchers have used two major model systems, Drosophila mushroom body neurons for axons and dendritic arborization (da) neurons for dendrites $[4,8,9]$. da neurons are born in the embryo, being constituent members of the peripheral nervous system, and generate two-dimensional dendrites underneath the epidermis and on the musculature during late embryonic and larval stages (Figure 1A) [10-12]. The 15 da neurons identified in each abdominal hemisegment are classified into four categories - classes I to IV - in order of increasing size of their receptive fields and/or arbor complexity at the mature larval stage (Figure 1B) $[8,12]$. In each hemisegment, the three class IV neurons develop the most complicated and expansive arbors with space-filling capability, whereas the three class I neurons generate sparse comb-like dendritic arbors [13,14]. The shaping of these class-specific dendritic arbors is controlled through the expression of a group of transcription factors in the post-mitotic neurons [15-21]. In the sense of physiological function, distinct subclasses appear to be responsible for sensation of proprioception and muscle contractions, which control larval crawling, and that of nociception, which protects larvae from parasitoid wasp attacks [2224].

It has been reported that a subset of da neurons persists during the pupal transition and that they exhibit largescale pruning and subsequent regeneration while keeping their axons superficially intact (Figure 1C-F) [25-28]. It is proposed that phagocytic blood cells scavenge neuronal debris and attack intact branches (Figure 1E; Additional file 1) [27]. Two out of the three larval class IV neurons in the hemisegment and one class I neuron, ddaE, are known to be survivors; the dendritic remodeling and migration of ddaE have been described $[26,29]$. At the molecular level, dendrite-specific pruning requires at least ecdysone signaling, ubiquitin-proteasomes, matrix metalloproteases, the I- $\kappa \mathrm{B}$ kinase-related kinase IK2, and a member of the Katanin family of microtubule-severing proteins [29-32]. Nevertheless, our knowledge regarding both the anatomical and molecular bases of the entire remodeling is still fragmentary. Although the soma of persistent larval sensory neurons have been identified [25], the dendritic arbor of each has not been described in the adult, and determination of the one-cell-to-one-cell relationship before and after the remodeling has not been completed either, except for the three neurons described above.

To establish the entire map of adult da neurons, we identified candidates of all survivors in pupae and adults, in both fixed dissected specimens and live whole-mount animals. We also genetically marked individual da neurons and traced the same cells at larval and adult stages. Altogether, our results identified six da neurons in the abdominal hemisegment 3 or $4(\mathrm{~A} 3 / 4)$ of the pharate adult and in the adult just after eclosion; all of these, except for one, were persistent larval da neurons. This 'baseline design' of A3/4 was modified in a segment-dependent and agedependent manner. Our study provides the anatomical and developmental bases for genetically hunting for mutations that affect remodeling of dendritic arbors or control of the life-long maintenance of differentiated neurons.

\section{Results}

Overview of peripheral neurons in dissected pharate adults With an expectation of identifying all da neurons in the abdomen of the pharate adult, we dissected animals of a transgenic line in which all larval da neurons are marked (Figure 2A,B; Gal4109(2)80 UAS-mCD8::GFP) [11,12] and stained them with anti-mCD8 or anti-green fluorescent protein (anti-GFP) antibody together with a pan-neuronal cell-surface marker, anti-horse radish peroxidase (antiHRP) antibody [33]. In these fillet preparations, the following three anatomical landmarks were helpful to map 

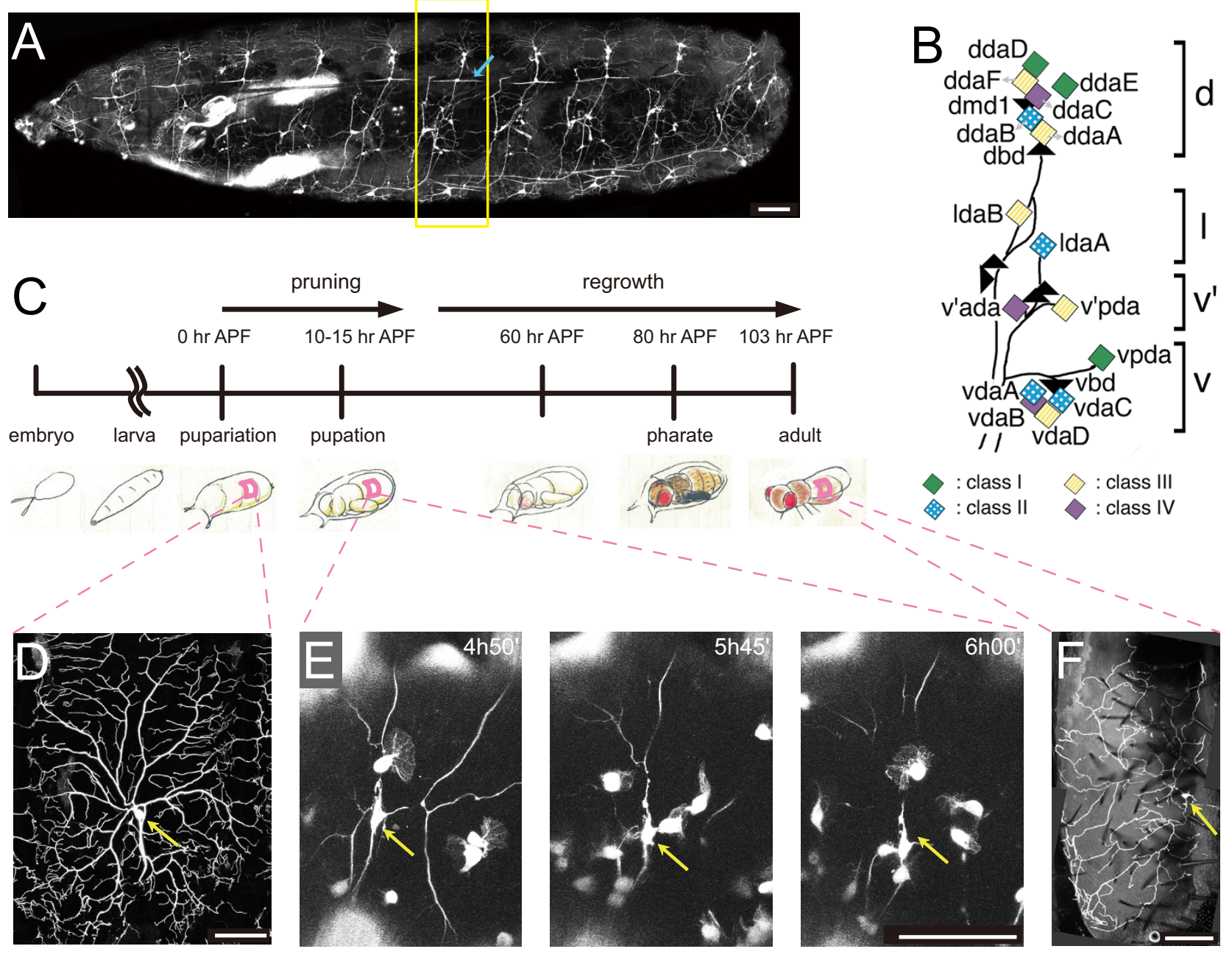

Figure I

Larval da neurons and timeline of dendritic remodeling during metamorphosis. (A) A lateral view of a third instar larva that highlights a large subset of dendritic arborization (da) neurons. Bipolar dendrites of dorsal bd (dbd) neurons are marked with blue arrows in this and subsequent figures. Genotype: UAS-mCD8::GFP/UAS-mCD8::GFP; CI6I UAS-mCD8::GFP/TM3 Ser Sb. Anterior is to the left, and dorsal is at the top throughout this study. (B) A diagram of positions of multidendritic (md) neurons in an abdominal hemisegment (A2 to A7) of the Drosophila larval peripheral nervous system. Diamonds represent individual da neurons, and triangles represent other types of md neurons. Of the other md types, names of two bipolar dendrite neurons (dbd and vbd) and dmd I are indicated for simplicity. $d, I, v '$, and v represent dorsal, lateral, ventral prime, and ventral clusters, respectively. Each class of the da neurons is differently colored. Black lines represent fascicles of axons that extend from the neuronal cell bodies. Adapted from [I7] with permission from Wiley-Blackwell. (C-F) Timeline (C) and representative images (D-F) of dendritic remodeling during metamorphosis. (C) $0 \mathrm{hr}$ after puparium formation (APF) indicates time of pupariation. (D-F) Images of class IV ddaC. The cell bodies are indicated by yellow arrows. (E) We visualized both ddaC and hemocytes and performed time-lapse recordings. Dendritic branches became detached from the cell body (arrow) and disappeared. During the pruning, the hemocytes migrated by generating prominent lamellipodia (see also Additional file I). (F) ddaC that regenerated its dendritic arbor in the adult tergite. Genotype: (D) ppk-EGFP/ppk-EGFP, (E) UAS-EGFP; ppk-EGFP/pxn-Gal4, and (F) FRTI9A/FRTI9A; Gal4109(2)80 UAS-mCD8::GFPI+. Scale bars: $100 \mu \mathrm{m}$ (A, D-F). 

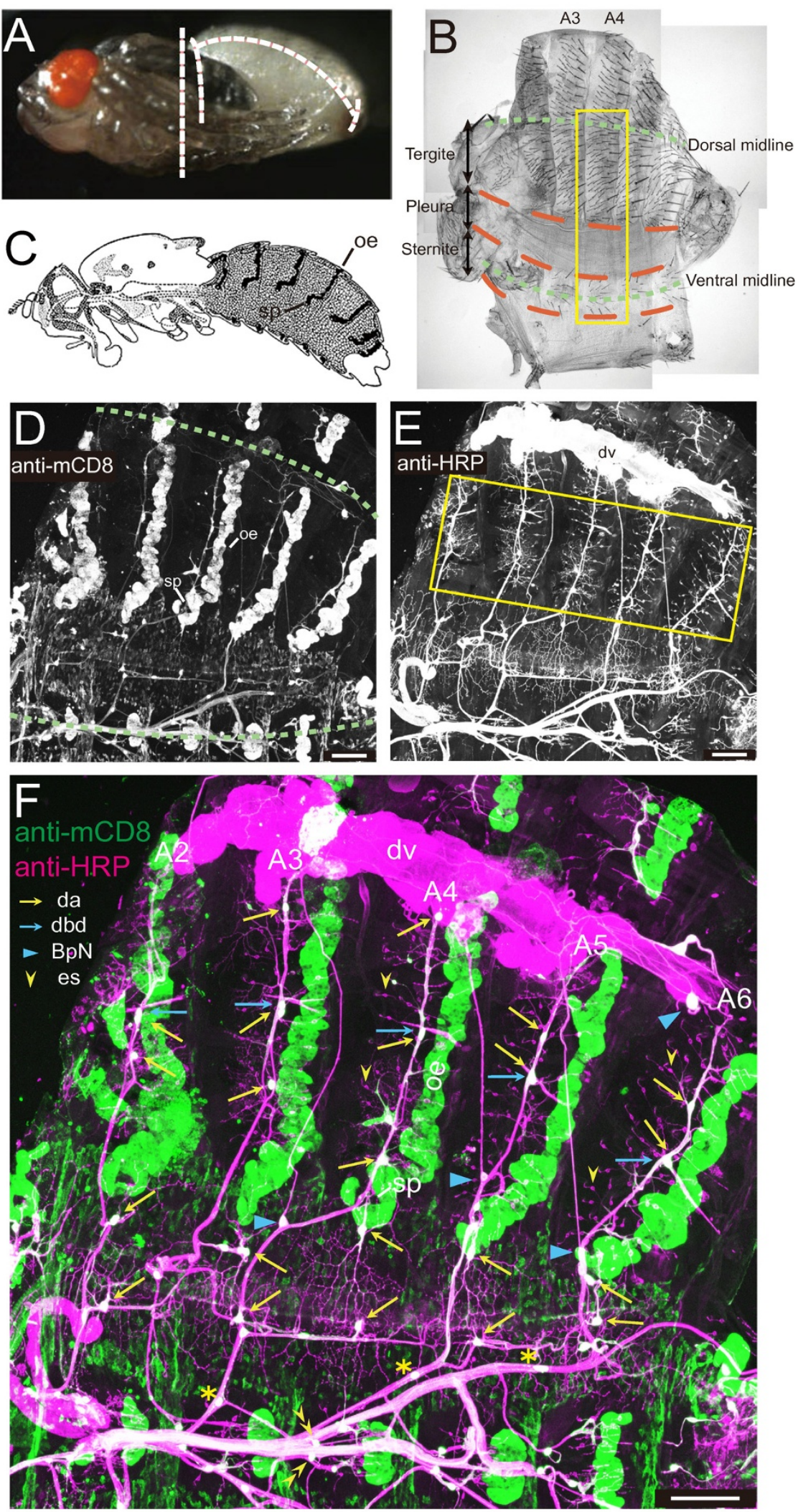

Figure 2 (see legend on next page) 
Figure 2 (see previous page)

Distribution of peripheral neurons in the abdomen of the pharate adult. (A) A lateral view of a pharate adult that was taken out of its puparium. Broken lines show how we dissected it. (B) A bright-field image of a fillet preparation of the abdomen of a male. Orange broken lines indicate boundaries between tergite, pleura, and sternite; green broken lines here and in (D) indicate dorsal and ventral midlines. The boxed region is highlighted in Figure 3. (C) Dark belts in the adult abdomen indicate oenocytes (oe). Adapted from FlyBase. sp, spiracle. (D-F) Double labeling of a fillet preparation of a male with antimCD8 antibody (D) (green in (F)) and a pan-neuronal marker, anti-HRP antibody (E) (magenta or white in (F)), of genotype Gal4109(2)80 UAS-mCD8::GFP/Gal4109(2)80 UAS-mCD8::GFP. dv, dorsal vessel (heart). The boxed region in (E) is highlighted in Additional file 4AA. In (F), A2 to A6 indicate numbers of abdominal segments. Yellow arrows point to identified dendritic arborization (da) neurons. Blue arrows indicate dorsal bd neurons (dbds), and blue arrowheads bipolar neurons that innervate the abdominal heart $(B p N)$ [34]. Yellow arrowheads point to representative external sensory (es) neurons, and double yellow arrowheads a pair of v'esB located on both sides of the ventral midline [25]. Asterisks indicate examples of mCD8::GFP-positive cells that were associated with fascicles. Scale bars: $50 \mu \mathrm{m}$.

candidates of adult da neurons in each segment: a spiracle at the tergite-pleura boundary (sp in Figure 2C,D,F); belts of oenocytes (oe in Figure 2C,D,F); and a bipolar dendrite (bd) neuron in the tergite that had been a dorsal bd ( $\mathrm{dbd}$ ) in larvae (blue arrows in Figure 2F) [26].

We examined which of mCD8::GFP HRP-double-positive cells extended long and/or complex dendrites, and designated those cells as da neurons at this developmental stage (yellow arrows in Figure 2F; high-power images are shown in Figure 3). In addition to dbd and da neurons, at least three other types of neurons existed in the periphery. First, monodendritic external sensory (es) neurons covered the tergite and the sternite (yellow single and double arrowheads in Figure 2F). Second, a bipolar neuron innervated the abdominal heart $(\mathrm{BpN}$ and blue arrowheads in Figure 2F) [34]. Third, a group of neurons was associated with peripheral axon fascicles in the sternite and the pleura (asterisks in Figure 2F).

\section{Six da neurons in hemisegment A3 or A4 of the pharate adult}

We observed the fillet preparations under high magnification, and traced dendrites and cell bodies of mCD $8:: \mathrm{GFP}$ HRP double-positive cells (Figure 3A-D). Aside from es, $\mathrm{dbd}$, bipolar, and the fascicle-associated neurons, all of the remaining HRP-positive cells also expressed mCD8::GFP; and they appeared to produce much longer and/or far more complex dendrites than es and dbd neurons. This finding suggests that Gal4109(2)80 UAS$m C D 8:: G F P$ marked all da neurons in pharate adults, as it does in larvae.

On the basis of the neuronal cell-surface staining, we found five da neurons in each of the abdominal hemisegments A3 and A4 (Figure 3D; the number of hemisegments examined was ten for each of A3 and A4). Our cellmarking experiment, which is described in Figure 4, clarified the origin of each neuron; aligned in the dorsal-toventral orientation were ddaD (larval class I), ddaC (larval class IV), ddaE (larval class I), ldaA (larval class II), and v'ada (larval class IV). Labeling neuronal nuclei, however, led us to notice that ldaA was associated with another neuron, ldaA-like (Figure 3D; see also Figure 5G-M; explained in Figure 6A-D). Thus, A3 or A4 had six da neurons in total, with three in the tergite and the other three in the pleura. Although it was difficult to distinguish dendritic branches of the individual neurons in this transgenic line, $\mathrm{ddaC}$ and v'ada appeared to develop expansive arbors as they did at the larval stage, whereas ddaE formed a bushy but much smaller one. Each ddaD extended possibly a single root dorsally. The dorsal-ventral position of ddaC relative to dbd was not fixed, and $\mathrm{ddaC}$ was sometimes located more ventral or attached to dbd. Besides the antiHRP antibody, we used monoclonal antibody $22 \mathrm{C} 10$ as another neuronal marker [35], which broadly labels embryonic and larval peripheral neurons; however, 22C10 stained cell bodies and axons but not dendrites of the da neurons in the pharate adult (data not shown).

With the above information from the fillet preparation in mind, we observed A3 or A4 in live whole-mount pupae of the pan-da marker line and those of marker lines for subsets of larval da neurons (Additional file 2). We found that markers for subsets of larval da neurons were not necessarily reliable tracers to pursue the relationship between larval da neurons and the pharate adult da cells (for example, see Additional file 2GG). We refer to the composition of da neurons in A3 and A4 of the pharate adult as 'the baseline design'. In subsequent sections, we describe origins of individual adult da neurons and their dendritic morphologies (Figures 4, 5 and 6; Additional file 3), expression in the pharate adult of transcription factors that endow larval class-selective dendritic morphologies (Figure 7), and also segment-dependent and age-dependent variations of this design (Figures 8 and 9; Additional file 4). 

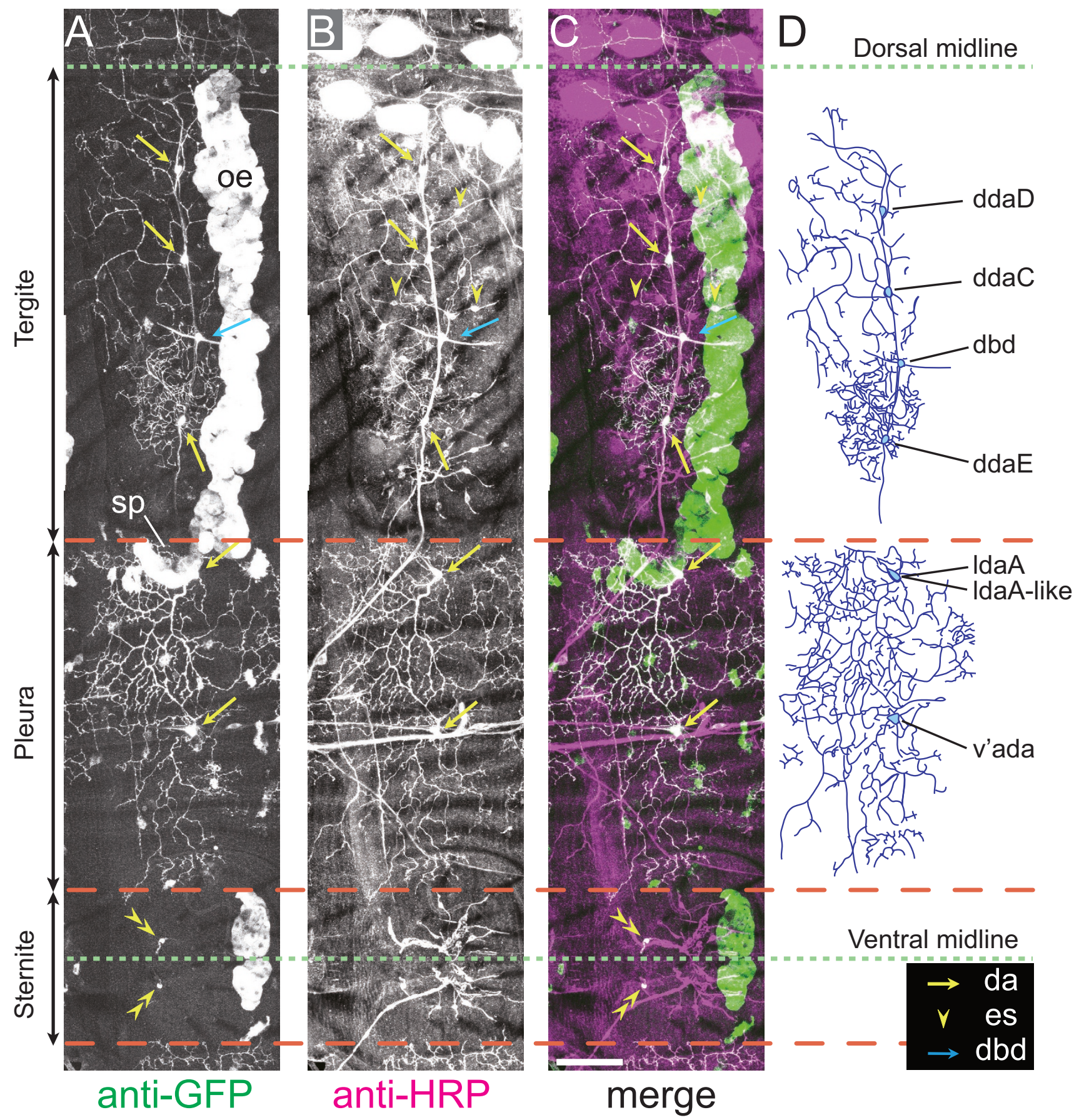

Figure 3 (see legend on next page) 
Figure 3 (see previous page)

Overall dendritic arbors of dendritic arborization (da) neurons in hemisegments A3 and A4 of the pharate

adult. A fillet preparation of a pharate adult male of genotype Gal4109(2)80 UAS-mCD8::GFP/Gal4109(2)80 UAS-mCD8::GFP was doubly stained with anti-GFP antibody (A) (green in (C)) and anti-HRP antibody (B) (magenta or white in (C)). (A-C) High-power immunohistochemical images of the boxed region in Figure 2B. (D) Dendrites, cell bodies, and axons of the da and dorsal bipolar dendrite ( $\mathrm{dbd}$ ) neurons were traced. We traced the origins of individual da neurons back to the larval stage (described later in Figure 4). IdaA and IdaA-like are a pair of closely associated cells (Figure 5G-M). The position of ddaC relative to dbd along the dorsal-ventral axis is not fixed (see text). Cell bodies of the da neurons were located more deeply than their dendrites. The pleura included GFP-positive, HRP-negative cells that had no obvious processes, and they were located more closely to the body surface than cell bodies of the da neurons. Identities of those cells are unknown. Scale bar: $50 \mu \mathrm{m}$. es, external sensory; oe, oenocytes; sp, spiracle.

\section{da neurons in adults}

We then addressed whether the baseline design at the pharate stage persisted in adults or not (Figure 5). Although tracing GFP-labeled dendritic branches in whole-mount adult abdomens was essentially as straightforward as in whole-mount larvae, the feasibility of visualizing branches in the adult specimens depended on sex, age, and whether the tracing was hindered by other intensely labeled cells, such as oenocytes, or not (Figure 5A,B). For example, branches were hardly visible in tergites of A5 and A6/A7 of males due to tanning of the cuticle as they aged (for example, see Figure $4 \mathrm{H}$ ). Nevertheless, ddaC appeared to form an expansive dendritic arbor; whereas ddaD seemed to have a single dorsally oriented root in A3 to A6 of both sexes, as they did in pharates (Figure 5C-F). To gain stronger membrane signals, some of our images were acquired in transgenic flies that expressed a fusion protein of mCD8 and three tandem copies of enhanced GFP (EGFP; Figure 5B). Co-labeling of membrane-bound GFP and nuclear proteins confirmed the close association of ldaA and ldaA-like (Figure 5I, its inset, and Figure 5K$\mathrm{M})$.

\section{Relationship between the larval and adult da neurons}

To trace the origins of the adult da neurons back to the larval stage, we labeled individual larval da neurons by the MARCM method [36] and tracked those cells up to the adult stage. More precisely, we first generated clones by using the pan-da driver Gal4109(2)80 or the pan-neuronal driver C155Gal4 (elav-Gal4), and recorded their cell identities and hemisegments where the clones were located in mature larvae. When the animals became adults, we mounted abdomens and scored all of the marked cells in observable segments as to whether each segment had possessed a clone at the larval stage or not, and attempted to match each of the adult da neurons to any of the larval ones (Figure 4; Table 1).

Our analysis revealed one-to-one relationships between four larval da neurons and the four adult ones. When 11 hemisegments that had produced the ddaD (class I) clones in larvae were tracked in adults, it was dorsally located neurons with single dorsally oriented roots that were marked in 11 out of the 11 hemisegments examined; and no such neuron was marked in any of the other hemisegments without ddaD clones (Figure 4B,G). Likewise, all clones of ddaC (class IV) and v'ada (class IV) were matched to neurons with expansive arbors in the tergite and those in the pleura, respectively; moreover, all of the larval ddaE clones were to neurons of small arbors with high branch density (see side-by-side pairs of panels in Figure 4 and numbers of clones examined in Table 1). No other larval da neurons contributed to these three labeled da neurons in the adult (Table 1). These results strongly suggest that each of the four larval da neurons underwent dendritic remodeling to become one of the four adult da neurons. Our results are consistent with reports that MARCM analysis using C161Gal4 shows the remodeling of ddaE [26]. Also, observations with the class IV-selective marker $p p k$-EGFP at distinct pupal stages show that daC and v'ada survive and they are designated as D neuron and $\mathrm{V}^{\prime}$ neuron, respectively [29] and that there are marked cells in the abdominal body wall of the adult [37].

When we tracked the six hemisegments that had produced ldaA (class II) clones in larvae, labeled in all of them in adults were neurons that were located close to the spiracles, with their dendritic arbors oriented ventrally (Figure $4 \mathrm{E}, \mathrm{J})$. In addition to the MARCM analysis, we labeled seven larval ldaA by a flipout-induced GFP expression and found that neurons with the above traits were marked in all of the seven hemisegments. Puzzlingly, we also found five hemisegments in which no larval da neurons had been marked by MARCM; but neurons with cellular locations and arbor shape similar to those of the adult ldaA showed up when the animals became adults. Because labeling of neuronal nuclei showed a pair of neurons to be tightly associated in the vicinity of the spiracle (Figure $5 \mathrm{G}-\mathrm{M}$ ), we speculate that larval ldaA became one of the two neurons in the adult and that the other adult neuron (ldaA-like) was derived from a cell that had not been identified as any of the larval da neurons. We attempted to label a pair of ldaA and ldaA-like in different colors by using the flipout-induced GFP expression in otherwise 

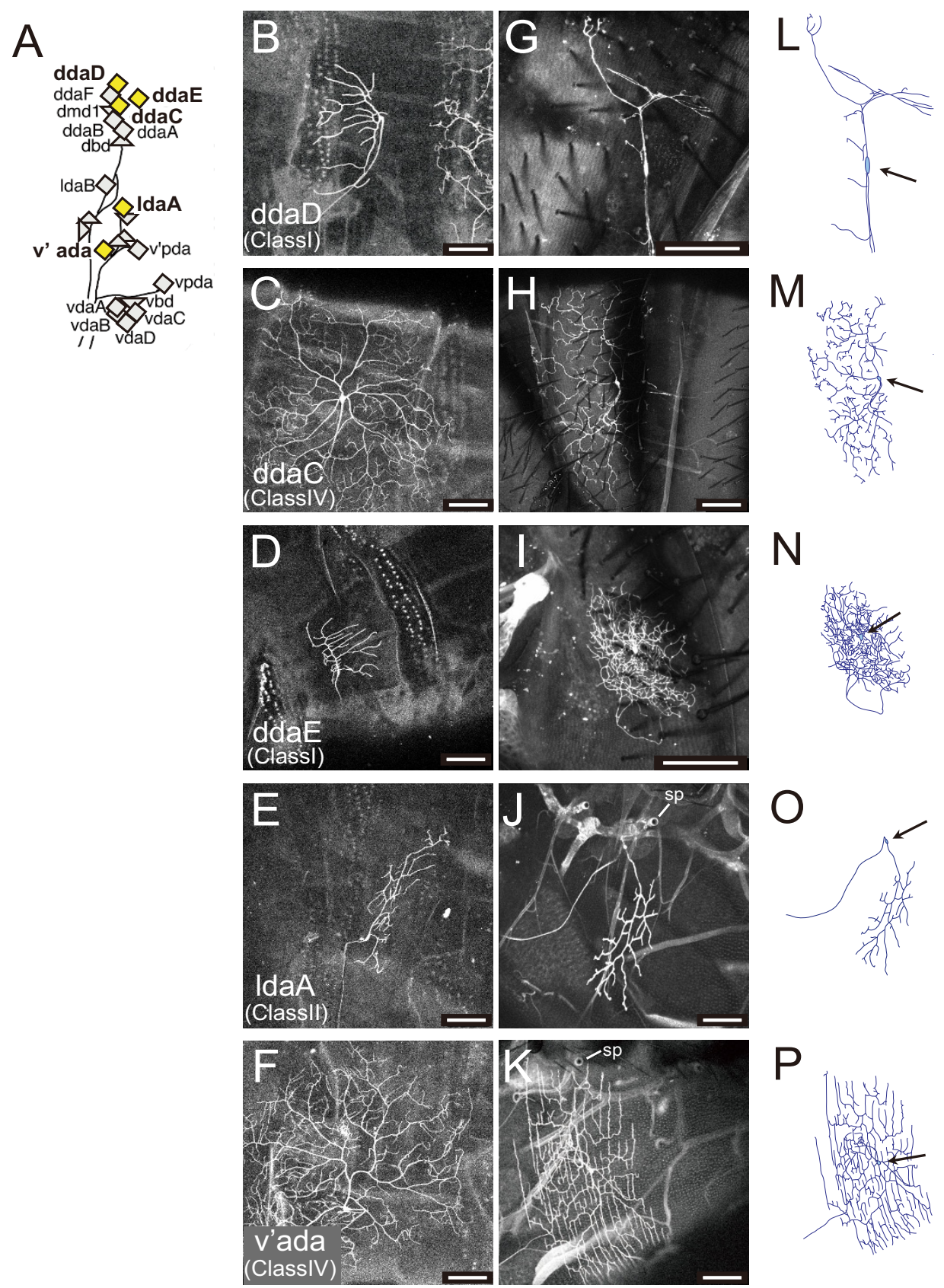

Figure 4

Relationship between a subset of larval dendritic arborization (da) neurons and the adult ddaD-v'ada. (A) A simplified version of Figure IB that highlights the five da neurons that survived metamorphosis (yellow diamonds). (B-K) Images of MARCM clones identified in third instar larvae (B-H) and of adult da neurons in the same hemisegments of the same animals that had generated the clones (G-K). Pairs of the larval and adult clones are shown side-by-side. Segments that produced clones were A4, A3, A2, A3, and A5 from the top. Genotypes of the clones: FRTI9A/FRTI9A; Gal4109(2)80 UAS$m C D 8:: G F P /+(C-F, H-K)$ or Gal4elav[CI55] UAS-mCD8::GFP hsflp/+; FRTG/3 L Sp/FRTG/3 L Sp (B, G). Images in (H, J, K) were acquired in 3- to 7-day-old adults, whereas those in (G, I) were of 0 - to 12-hour-old adults. sp, spiracle. (L-P) Tracings of (GK). Arrows point to cell bodies. Note that the adult da neurons (G-P) are shown with various scales and that $\mathrm{ddaC}(\mathrm{H}, \mathrm{M})$ and v'ada $(K, P)$ formed much more expansive arbors than the other neurons. Scale bars: $50 \mu \mathrm{m}$. 

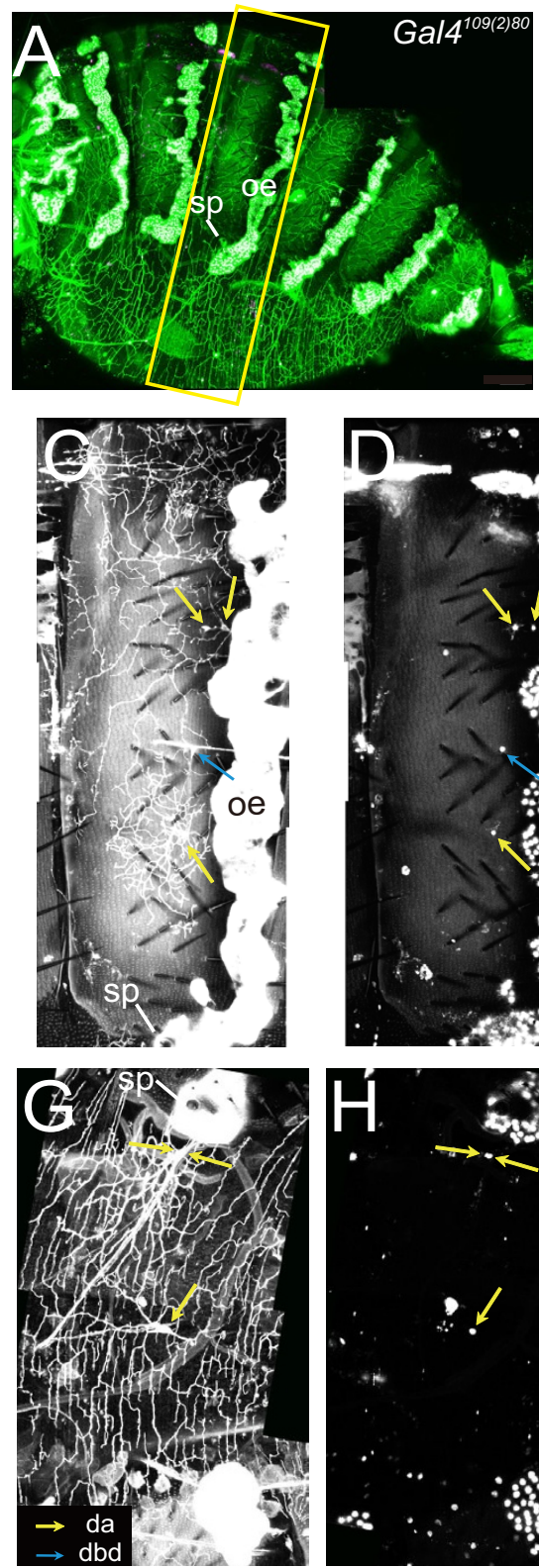

mCD8::GFP
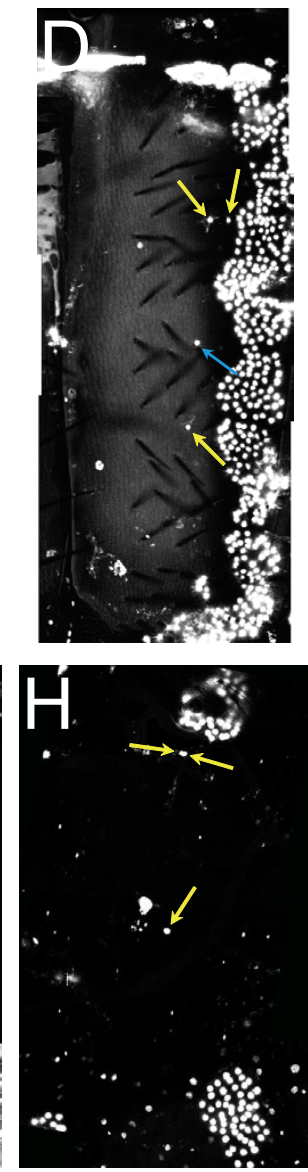

RedStinger
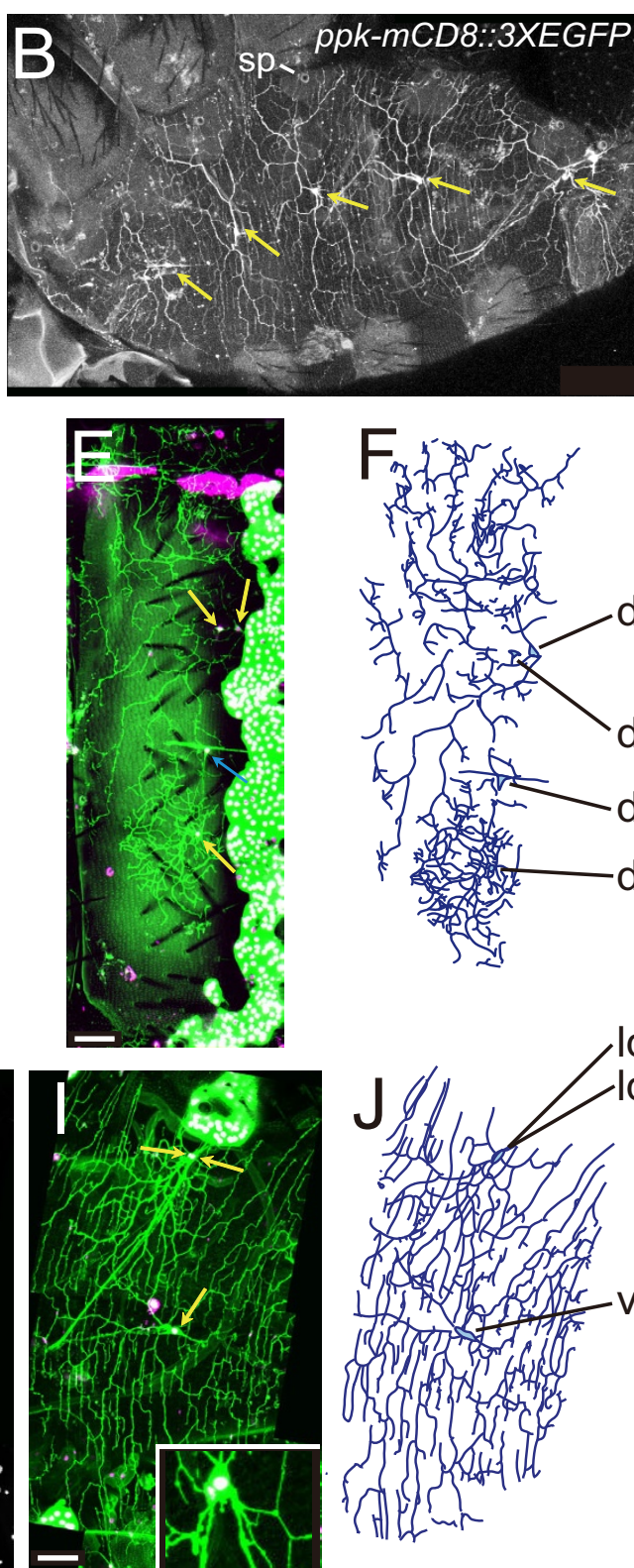

merge

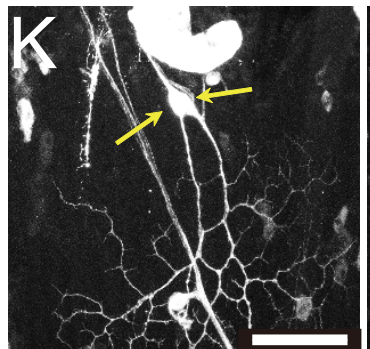

anti-mCD8

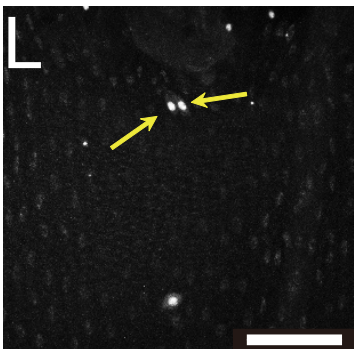

anti-Elav
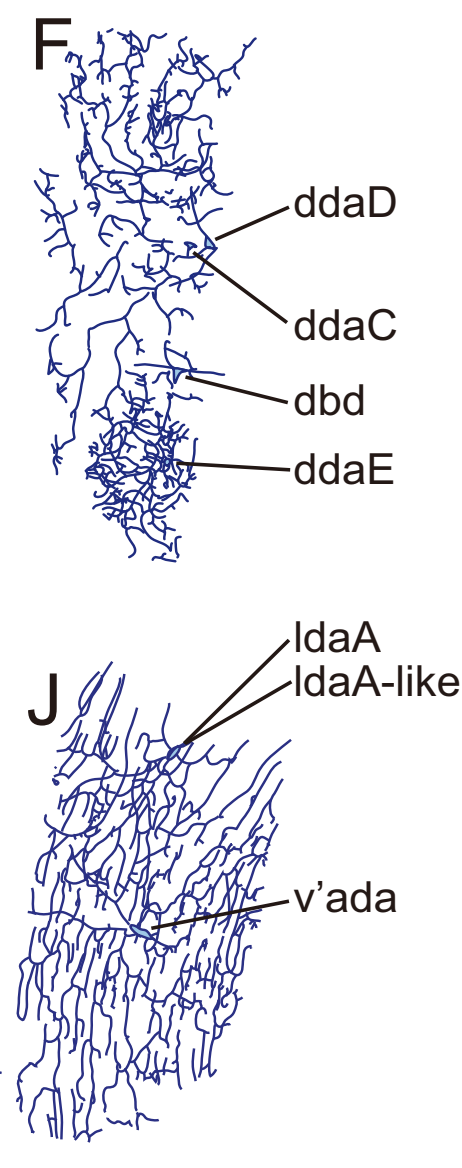

merge

Figure 5 (see legend on next page) 
Figure 5 (see previous page)

Dendritic arborization (da) neurons in the adult. (A) A lateral image of a whole-mount abdomen of a I- to 3-day-old female of genotype UAS-RedStinger/+; Gal4109(2)80 UAS-mCD8::GFP/+. RedStinger encodes a nuclear targeted DsRed. The yellow rectangle indicates A4. oe, oenocyte; sp, spiracle. (B) A ventral-lateral image of the pleura of a 3-to 4-day-old male of ppk$m C D 8:: 3 x E G F P / p p k-m C D 8:: 3 x E G F P$. The pleura was covered with lattice-shaped dendritic arbors of v'ada neurons (yellow arrows), and a couple of branches in each arbor were more strongly labeled than the others. (C-J) High-power images of the tergite (C-E) and those of the pleura and the sternite (G-I) in A3/A4 of another whole-mount female of the same genotype as in (A). The channel signals of GFP (C, G) (green in (E, I)) and DsRed (D, H) (magenta or white in (E, I)) are shown. Yellow arrows point to nuclei of da neurons, and blue ones to those of dorsal bipolar dendrite (dbd) neurons. Nuclei of IdaA and IdaA-like are closely associated with each other (G-I; the inset in (I) shows a higher-power image of a different hemisegment). $(F, J)$ Tracings of dendrites and cell bodies in $(C)$ and $(G)$, respectively. (K-M) A fillet preparation of a pharate adult female of genotype Gal4109(2)80 UAS-mCD8::GFP/Gal4109(2)80 UAS-mCD8::GFP. mCD8 staining (K) (green in (M)) and Elav staining (L) (white in $(\mathrm{M})$ ), and a merged image (M). This high-power image highlights a pair of nuclei of IdaA and IdaA-like. Scale bars: $50 \mu \mathrm{m}(\mathrm{c}-$ $\mathrm{m})$.

CD2-expressing neurons in the adult (Figure 6A-D). This labeling method showed that the cell bodies of these two neurons were attached to each other and that their dendritic arbors were substantially overlapped, as we had expected (number of pairs labeled $=3$ ).

\section{Morphological features of dendritic arbors of the adult da neurons}

To understand the morphological features of individual arbors, we analyzed the arbor shape of clones identified in adults. To compare arbors of ldaA neurons with those of ldaA-like ones quantitatively, we traced the arbors of the two neuronal types and measured the total branch length of each type (Figure 6E-G). Dendritic arbors of the two neurons did not differ significantly in terms of total branch length. Intriguingly, v'ada, which had extended dendritic branches in a radial fashion at the pharate stage and just after eclosion, reshaped its arbor into a lattice pattern during post-emergence growth (Figure 6H,I). This radial-to-lattice transformation was recognizable in 1day-old adults, and most terminal branches tended to be oriented along the dorsal-ventral axis of the body. All of the v'ada clones observed from day 1 onwards elaborated lattice-shaped arbors (Figure 6J). We were able to measure total branch length of the v'ada clones at different adult ages and found that it did not alter during aging (Figure $6 \mathrm{~K})$, suggesting that the arbor of v'ada was maintained during adult life. The total branch length of adult v'ada of A4/5 was around $10 \mathrm{~mm}$ (Figure 6K), whereas that of the same cell type in the same hemisegments in the wandering third instar was around $15 \mathrm{~mm}$ (data not shown).

Compared to ddaC and v'ada arbors, ddaE arbors were smaller in adults (Figure 5F; also compare the scale bar of Figure $4 \mathrm{I}$ with that of Figure $4 \mathrm{H}, \mathrm{K}$ ) as they were in larvae [12]. The terminal number of ddaE was remarkably increased after the remodeling, as shown by the fact that each larval ddaE has only about 30 terminals [21], whereas the adult ddaE had over 100 terminals. Appar- ently, ddaD arbors were the least stereotypic; cell bodies extended single branches dorsally, and they sprouted in a variable fashion (Additional file $3 \mathrm{AA}$ ). ddaD branches in A3/A4 tended to be longer than those in A5/A6 and to grow along the dorsal vessel.

In addition to the morphological analysis of the clones, we surveyed various Gal4 lines for their expression patterns in the adult (Table 2). Some of those lines will be helpful to visualize dendritic arbors at single-cell resolution, to quantify their morphological features, and to analyze physiological functions of the labeled neurons in the future. For example, adult ldaA or ldaA-like was marked by Gr66aGal4, which is a fusion of the taste receptor gene Gr66a and Gal4 (Additional file 3BB) [38], and the same neuron was labeled also by en-Gal4 (data not shown; Figure 7D in [39]). Neither Gr66aGal4 nor en-Gal4 labeled larval ldaA, so we could not conclude whether the labeled neuron in the adult was ldaA or ldaA-like. Gr66a is one of the Drosophila caffeine receptors [40,41], and Gr66aGal4 labels gustatory receptor neurons that detect aversive chemicals [42].

\section{Was the dendritic remodeling associated with selective expression of the shape-controlling transcription factors?} It has been shown in larvae that shaping of class-specific dendritic arbors is controlled by a group of transcription factors in the post-mitotic neurons and that some of these factors are expressed in a class-selective or class-differential manner [15-18,20,21]. Knot (Kn)/Collier of the early $\mathrm{B}$ cell factor family is expressed selectively in all of the class IV neurons in the embryo, and it is essential for generation of their complicated and expansive arbors $[15,17,18]$. Its immunohistochemical signal at the mature larval stage was very weak or hardly detected in the class IV neurons (data not shown). Interestingly, in pharate adults Kn expression was revived in both of the two surviving and regrowing class IV neurons (six out of seven $\mathrm{ddaC}$ and nine out of nine v'ada examined); on the other 


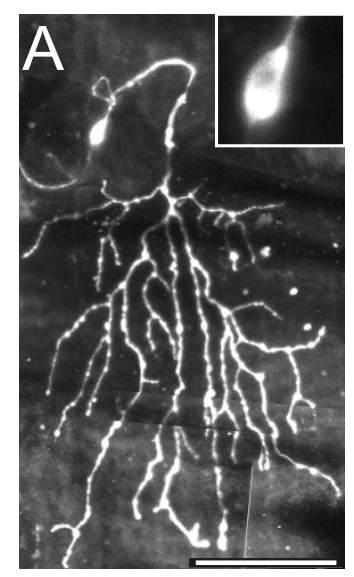

anti-GFP

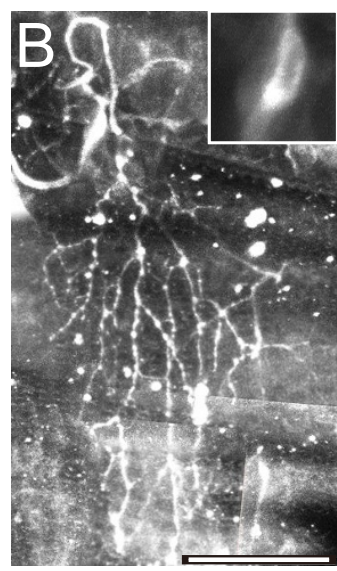

anti-CD2

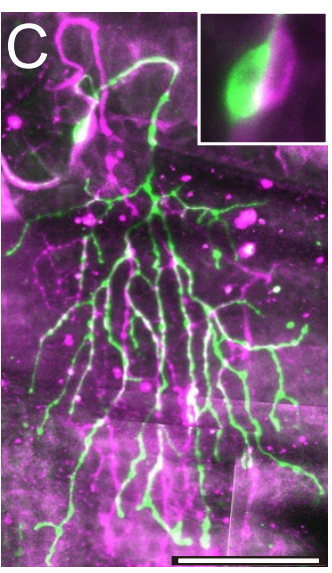

merge
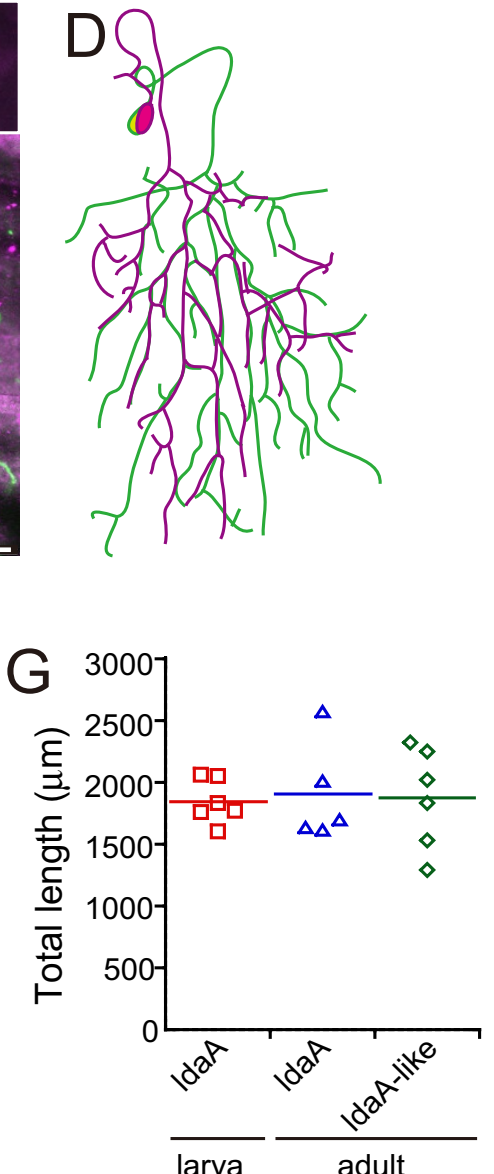

IdaA-like
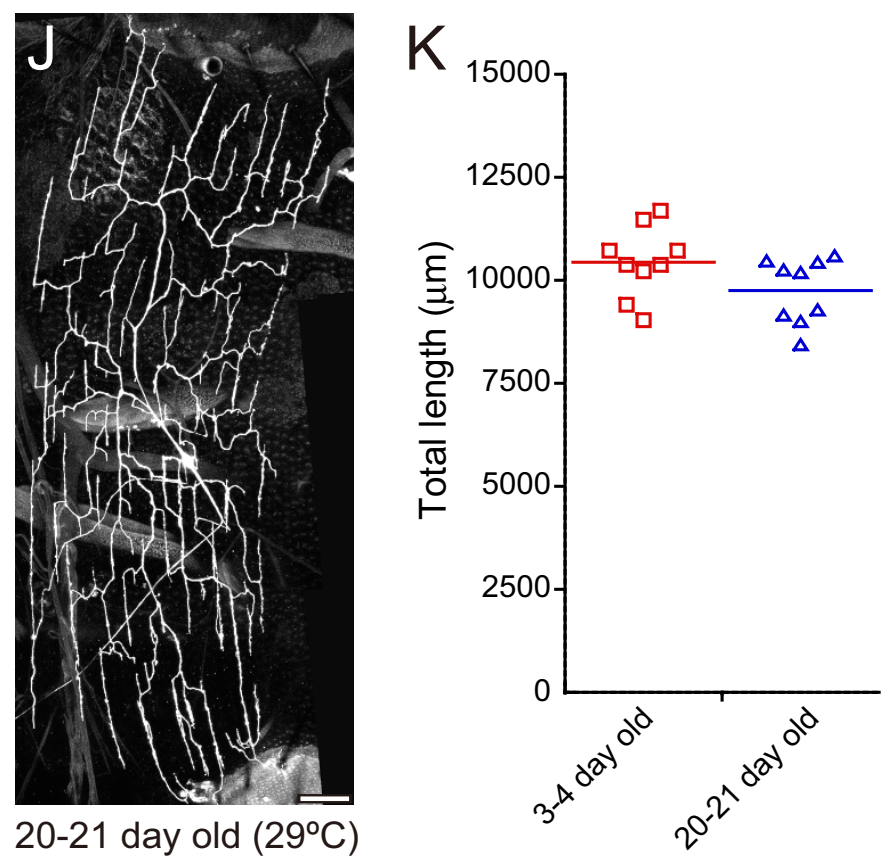

Figure 6 (see legend on next page) 
Figure 6 (see previous page)

Morphological features of dendritic arbors of IdaA/IdaA-like and v'ada neurons. (A-D) A pair of IdaA and IdaA-like neurons labeled in different colors by using GFP antibody to visualize the flipout-induced GFP expression (A) (green in (C)) in otherwise CD2-expressing neurons (B) (magenta or white in $(C)$ ) in the adult. A merged image (C) and tracings (D) are also shown. Insets indicate high-power images of cell bodies. We did not observe this hemisegment of this animal at a larval stage, so we could not conclude which neuron was derived from larval IdaA. Genotype: $y w$ hsflp/+;UAS-FRT-CD2,y+-FRT-mCD8::GFP/+; $\mathrm{CI} 6 \mathrm{I} / \mathrm{TM} 2$. (E-G) Comparison of arbors of IdaA and IdaA-like neurons. Tracings of representative adult clones of IdaA (E) and IdaA-like (F) in A2 to A5 are shown. These neurons in (E) were designated as IdaA in the adult or simply as 'adult IdaA' because the same hemisegments of individual animals had produced IdaA clones at the larval stage. See the definition of 'IdaA-like' in the text. (G) Plots of total length of dendritic branches of individual larval IdaA (red squares), adult IdaA (blue triangles), and IdaAlike (green diamonds). Horizontal bars indicate means. (H-K) MARCM clones of v'ada in A2 to A5 were found, and those adult flies were aged at $29^{\circ} \mathrm{C}$. Neurons at 0 to 6 hours $(\mathrm{H}), 3$ to 4 days $(\mathrm{I})$, and 20 to $2 \mathrm{I}$ days $(\mathrm{J})$ after eclosion are shown. The clones were generated as described in the legend of Figure 4. (K) Total length of dendritic branches of individual v'ada arbors of A4 and $\mathrm{A} 5$ at 3 to 4 days (red squares) and at 20 to 21 days (blue triangles). Horizontal bars indicate means. Scale bars: $50 \mu \mathrm{m}$.

hand, Kn expression was detected in no other da neurons at this stage (Figure 7A-F). A homeo-domain protein Cut (Ct) is differentially expressed in distinct subclasses of embryonic and larval da neurons, with the highest level in class III neurons and an intermediate level in class IV neurons [16]. In pharate adults in which larval class III neurons had not been found, we detected only a weak level of Ct expression in the class IV neurons (three out of three $\mathrm{ddaC}$ and six out of six v'ada), and none in any other of the da neurons (Figure 7G-L). It should be noted that the $\mathrm{Kn}$ or $\mathrm{Ct}$ proteins seemed to be distributed in both the nucleus and cytoplasm (see insets of Figure 7), which is in contrast to the almost exclusive nuclear localization in the embryo [15-18]. The simple comb-like dendritic arbor of larval class I neurons ( $\mathrm{ddaD}$ and $\mathrm{ddaE}$ ) is endowed by expression of the BTB-zinc finger protein Abrupt (Ab) $[20,21]$. Ab was expressed in ddaE in mature larvae, whereas the expression was no longer detected in the same cell type when it had remodeled into a highly bushy arbor in the pharate adult (data not shown; number of hemisegments examined $=4$ ). Compared with that in ddaE, Ab expression in ddaD was already weak or hardly detected in mature larvae, and the expression remained off in the pharate adults (data not shown).

\section{Segment-dependent variations in the composition of da neurons}

We found that the A3/A4 baseline design of the da neurons in the tergite was modified in a segment-dependent manner. One obvious difference between segments was that ddaE was invisible in A5 and in more posterior segments in several Gal4 lines examined (Figure 8A; see also Figure 9A). Because ddaE is present in all larval A2 to A6 segments, we postulated that ddaE in A5 and A6 had either died during metamorphosis or ceased GFP expression. To distinguish these possibilities, we examined A2 to A6 by staining them with two Gal4-independent pan-neuronal markers (Additional file 4). Our results strongly suggest that ddaE was absent in $\mathrm{A} 5$ and $\mathrm{A} 6$ in the pharate adult. Cell death of ddaE in those segments was verified by our time-lapse recordings (Figure 8B-G; Additional file $5)$. In A5, a ddaE-like cell started re-growth of its dendrites after the pruning phase (Figure $8 \mathrm{~B}$ ) and then degenerated (Figure $8 \mathrm{C}$ ). Our staining of neuronal nuclei suggested the composition of da neurons in each segment at the pharate stage (Additional file 4GG).

We could find ddaE neurons in A2 to A4, which had survived pupal development, in 0 - to 1 -hour adults with complete penetrance (Figure 9A,D); however they became hardly seen by 1 week after eclosion (Figure 9B,D). With the help of the pan-neuronal nuclear marker Elav, we confirmed this loss of ddaE by scoring neuronal nuclei of multidendritic neurons (da plus bipolar dendrite) in dissected adults at the age of 0 or 7 days (Figure 9E). Expression of the caspase inhibitor p35 kept ddaE in A2 to A4 alive, suggesting that ddaE underwent caspase-dependent cell death (Figure 9C,D). In contrast to dendritic arbors of ddaE, those of ddaC, ldaA, and v'ada were visible in transgenic flies over 3 weeks old, even during their entire adult life (data not shown).

\section{Discussion \\ Comparisons with previous reports regarding origins of pupal and adult multidendritic sensory organs}

In our study, we identified all multidendritic neurons in the abdomens of the pharate adult and the adult, visualized dendritic arbors of the individual neurons, and attempted to clarify their larval origins. Specifically, we revealed one-to-one relationships between the five larval da neurons and five out of the six da neurons identified in the adult abdomen. Our conclusion regarding ddaC, ddaE, and v'ada agrees with two previous reports that describe those neurons at pupal stages [26,29]. It is reported that larval class I ddaD escapes death and that its dendrites are pruned in prepupa [27]; however, its subsequent fate through to the adult stage was not described. Our result shows that the most dorsally located da neuron 

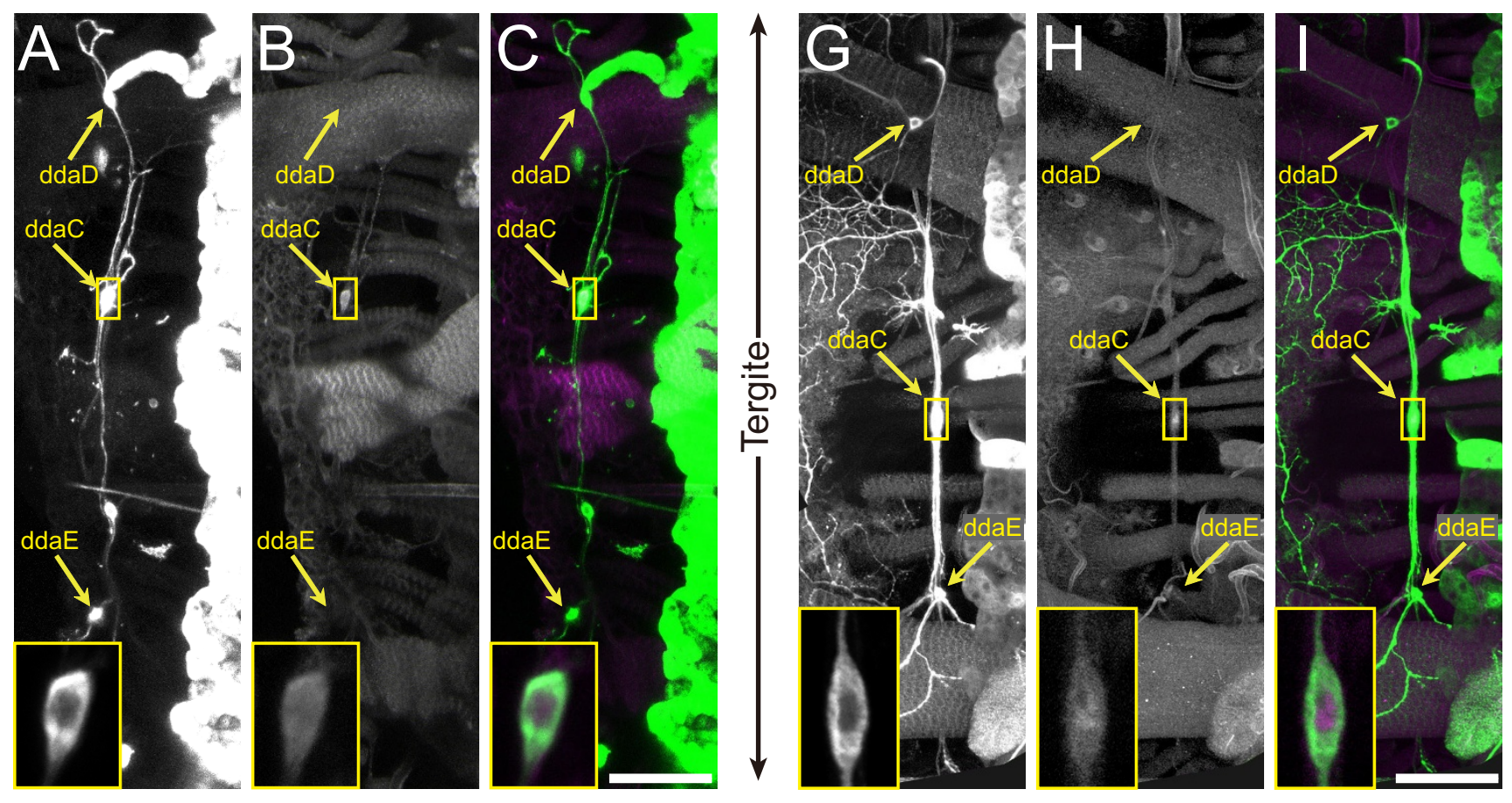

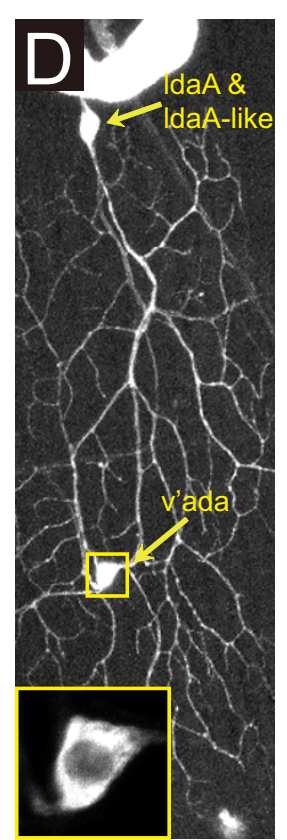

anti-GFP

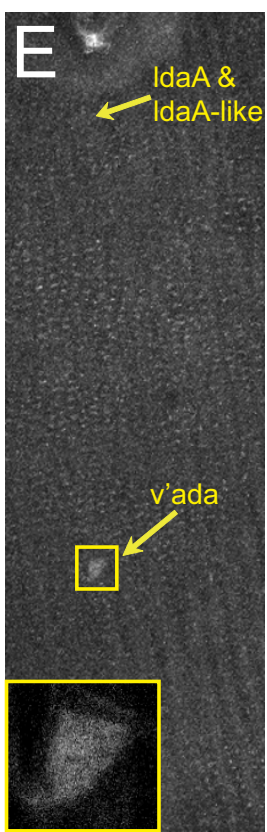

anti-Knot

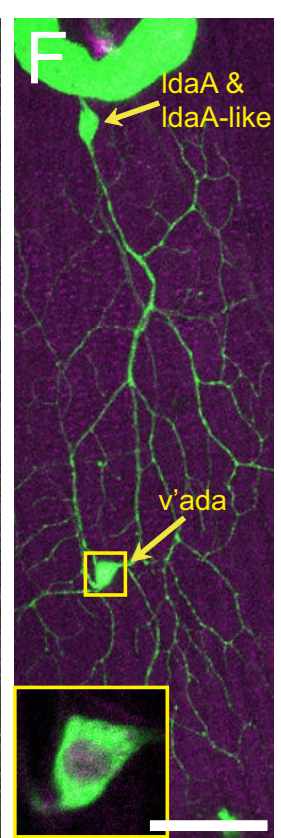

merge

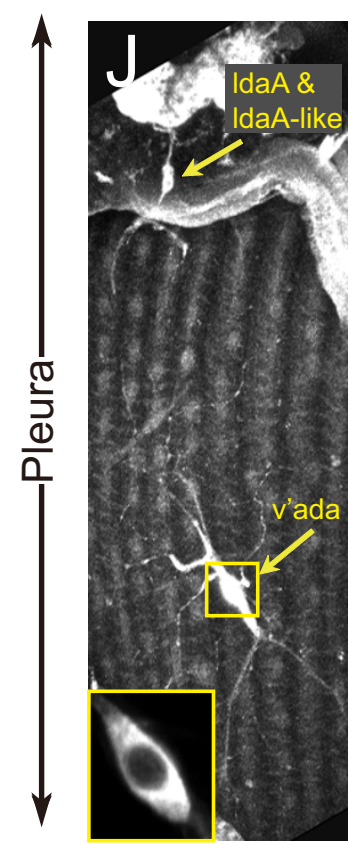

anti-GFP

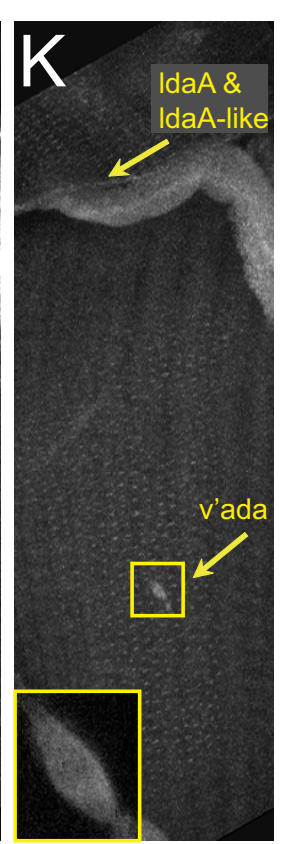

anti-Cut

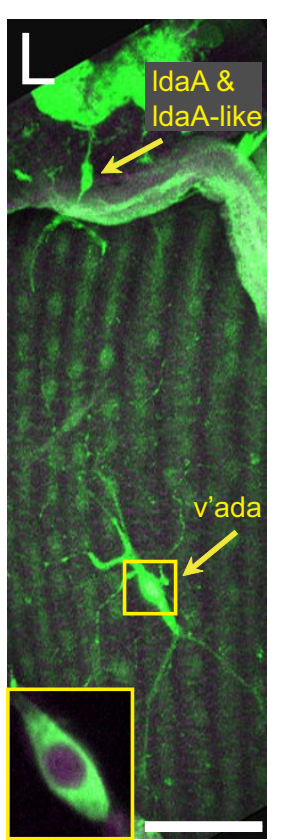

merge

Figure 7

Expression of shape-controlling transcription factors in pharate adults. Fillet preparations of pharate adults were doubly stained with anti-GFP antibody and antibody against transcription factors. Yellow arrows point to cell bodies of individual dendritic arborization (da) neurons. Cell bodies of ddaC and v'ada are highlighted in the insets. Genotype: Gal4109(2)80 UASmCD8::GFP/+. (A-F) Neurons stained for GFP (A, D) (green in (C, F)) and for Knot (B, E) (magenta or white in (C, F)). (G-L) Neurons stained for GFP (G, J) (green in $(\mathrm{I}, \mathrm{L})$ ) and for Cut $(\mathrm{H}, \mathrm{K})$ (magenta or white in $(\mathrm{I}, \mathrm{L})$ ). Scale bars: $50 \mu \mathrm{m}$. 

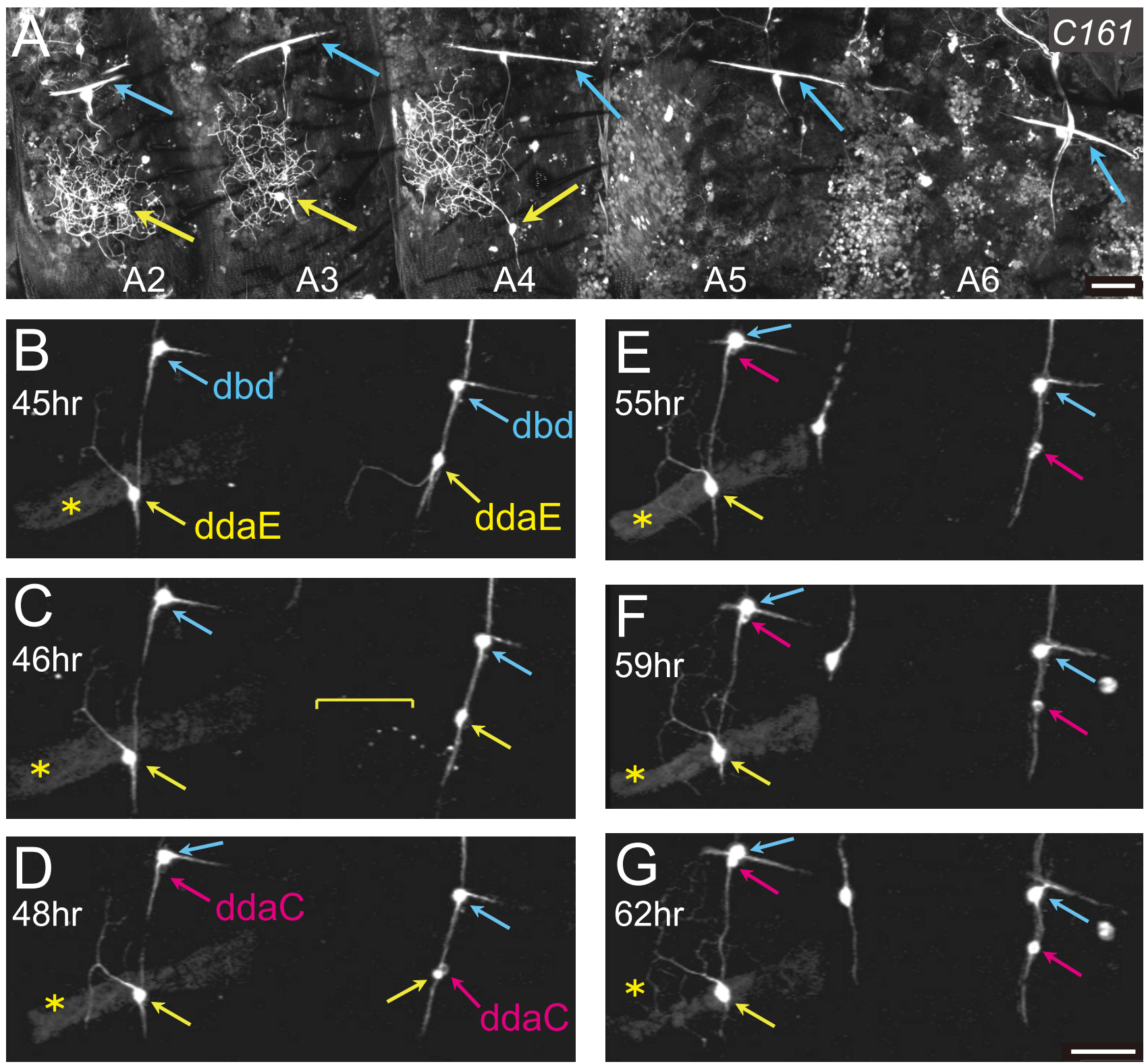

\section{Figure 8}

ddaE in A5 started regeneration of dendrites and then degenerated. (A) A whole-mount of a pharate adult female of genotype UAS-mCD8::GFP/+; CI6/Gal4/+. Yellow arrows in A2 to A4 point to cell bodies of ddaE, and blue arrows to dendrites of dorsal bipolar dendrite (dbd) neurons. (B-G) Time-lapse recordings of a tergite of A4 and A5 of genotype UAS-mCD8::GFPI UAS-mCD8::GFP; CI 6 I UAS-mCD8::GFP/TM3 Ser Sb from 45 h after puparium formation (APF) (B) to 62 h APF (G). Yellow, magenta, and blue arrows indicate $\mathrm{ddaE}, \mathrm{ddaC}$, and dbd, respectively. $\mathrm{A} 4$ and $\mathrm{A} 5$ were identified by the respective presence and absence of a larval persisting muscle (the dorsal internal oblique muscle 3 (DIOM3); yellow asterisks) [43]. A branch of $\mathrm{ddaE}$ in A5 was degraded at $46 \mathrm{~h}$ APF (yellow bracket in (C)), and then the cell body started condensation (right yellow arrow in (D); see also Additional file 5). Scale bars: $50 \mu \mathrm{m}$ (A, B-G). 

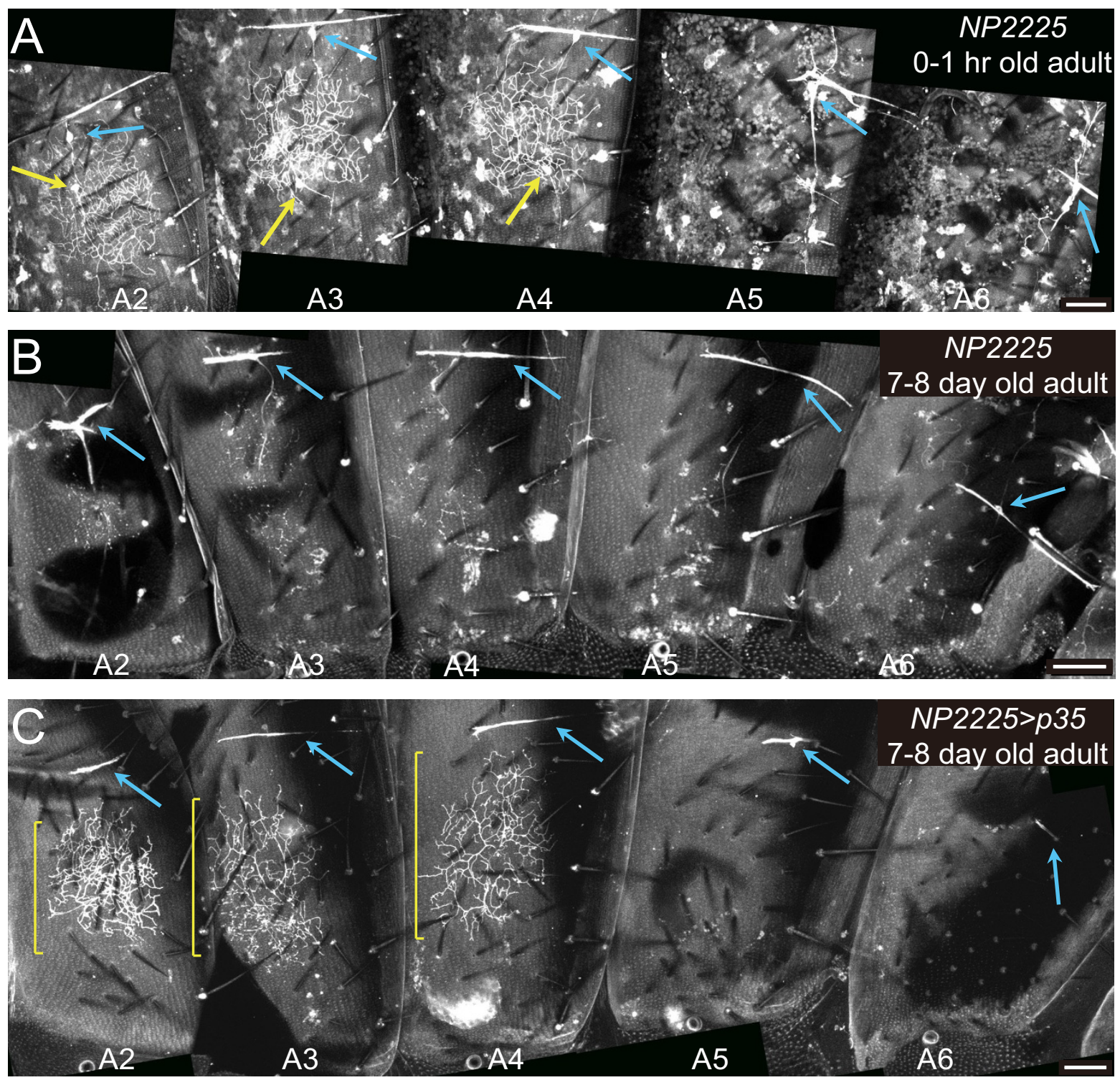
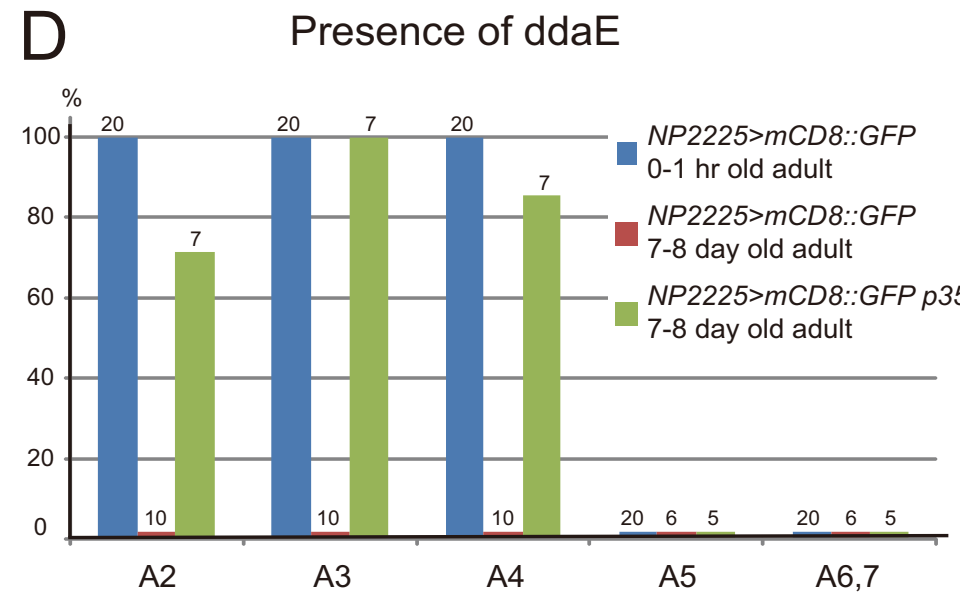

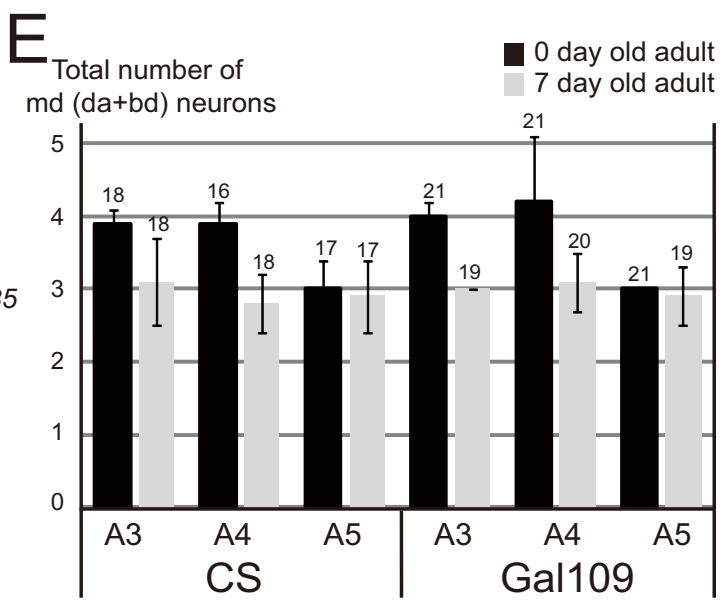

Figure 9 (see legend on next page) 
Figure 9 (see previous page)

Almost all ddaE neurons underwent apoptosis within I week after eclosion. (A-C) Whole-mounts of a 0- to Ihour-old female of genotype NP2225 UAS-mCD8::GFP/CyO (A), a 7- to 8-day-old female of the same genotype (B), and a 7- to 8day-old female of genotype NP2225 UAS-mCD8::GFP/CyO; UAS-p35/+ (C). Yellow arrows in (A) point to cell bodies of ddaE in $A 2$ to $A 4$, and blue arrows in (A-C) to those of dorsal bipolar dendrite (dbd) neurons. Yellow brackets in (C) indicate dendritic arbors of surviving ddaE. Scale bars: $50 \mu \mathrm{m}$. (D) The percentage of each hemisegment that had ddaE. Genotypes and ages of male adults examined are indicated, and the number of each hemisegment observed is given above each bar. Colored short lines just on the $x$-axis mean that no ddaE signal was found in the hemisegment examined. (E) Quantification of the total number of multidendritic (md) neurons (da plus dbd) in each tergite of Canton S (CS) and Gal4109(2)80 UAS-mCD8::GFP/ Gal4109(2)80 UAS-mCD8::GFP (Gal 109) in 0- or 7-day-old adults. Adults were dissected and stained for HRP and Elav as in Additional file 4B-FB-F; and the number of md neurons in each tergite was counted. Each value is expressed as mean \pm standard deviation. The number of animals examined is indicated above each bar.

with a single dendritic root in the adult abdomen was derived from larval ddaD.

In addition to those studies cited above, embryonically derived sensory neurons are identifiable at pupal and pharate adult stages by using a lineage tracer, although this cell-labeling method does not visualize entire dendritic arbors [25]. That paper reported that class II ldaA is one of the persistent larval sensory neurons, which is consistent with our result. The above paper also describes that class III v'pda is a persistent neuron; however, we found that none of the ten v'pda larval clones persisted in the adult (Table 1) and that it was class IV v'ada in the same v' cluster that survives metamorphosis.

\section{Segmental differences in the timing of programmed cell death}

ddaE neurons in all of the abdominal hemisegments executed programmed cell death (PCD) at pupa to young adult stages, and they showed segmental differences in the execution time window. ddaE in A2 to A4 died within 1 week after eclosion, whereas PCD of ddaE in the more posterior segments took place at the midpupal stage. It should be noted that this segmental difference in the PCD is correlated with the spatiotemporal profile of the breakdown of dorsal internal oblique muscle (DIOM)3, on which ddaE is located (asterisk in Figure 8B-G) [43]. DIOM3 in A5 to A6 dies at 16 to 20 hours after puparium formation (APF), whereas the PCD of DIOM3 in A1 to A4 takes place within 1 day after eclosion. It remains to be

Table I: MARCM analysis to trace the origins of adult dendritic arborization neurons back to the larval stage.

\begin{tabular}{|c|c|c|c|}
\hline & & Number of clones in third instar larvae & Number of clones in the adults \\
\hline \multirow[t]{6}{*}{ d } & ddaA (class III) & 9 & 0 \\
\hline & ddaB (class II) & 8 & 0 \\
\hline & $\mathrm{ddaC}$ (class IV) & 19 & 19 \\
\hline & ddaD (class I) & 11 & $1 I^{a}$ \\
\hline & ddaE (class I) & 3 & $3^{\mathrm{a}}$ \\
\hline & ddaF (class III) & 21 & 0 \\
\hline \multirow[t]{2}{*}{ I } & IdaA (class II) & 6 & 6 \\
\hline & IdaB (class III) & 9 & $\mathrm{I}^{\mathrm{b}}$ \\
\hline \multirow[t]{2}{*}{$v^{\prime}$} & v'ada (class IV) & 10 & 10 \\
\hline & v'pda (class III) & 10 & 0 \\
\hline \multirow[t]{5}{*}{$\mathrm{v}$} & vpda (class I) & 6 & 0 \\
\hline & vdaA (class II) & 3 & 0 \\
\hline & vdaB (class IV) & 4 & 0 \\
\hline & $\mathrm{vdaC}$ (class II) & 4 & lb \\
\hline & vdaD (class III) & 2 & 0 \\
\hline
\end{tabular}

We first generated MARCM clones, and recorded their cell identities and hemisegments where the clones were located in mature larvae. When the animals became adults, we mounted their abdomens and scored all of the marked cells in the recorded hemisegments. We excluded from counting all larvae in which multiple clones were marked in single hemisegments. d, I, v', and v represent dorsal, lateral, ventral prime, and ventral clusters, respectively. 'Clones were identified within 12 hours after eclosion. This was partly because Gal4109(2)80-dependent GFP signals were difficult to find in ddaD at later stages, and partly because ddaE executed programmed cell death within I week after eclosion. bWe interpreted this clone to be IdaA-like that most likely had been derived from a cell other than larval dendritic arborization neurons (see details in the text). 
Table 2: Expression patterns of Gal4 drivers in the adult Drosophila abdomen (A2 to A6).

\begin{tabular}{|c|c|c|c|c|c|c|}
\hline \multirow[b]{2}{*}{ Genotype } & \multicolumn{3}{|c|}{ Tergite } & \multicolumn{3}{|c|}{ Pleura } \\
\hline & ddaD & ddaC & ddaE & IdaA, IdaA-like & v'ada & Reference \\
\hline Gal4 [4-77] UAS-mCD8::GFP & + & + & + & + & + & {$[16]$} \\
\hline I003.3Gal4/UAS-mCD8::GFP & $?$ & + & $?$ & + & + & {$[22]$} \\
\hline Gal4 [5-40]/+; UAS-mCD8::GFP/+ & + & + & + & + & + & [24] \\
\hline CI55 UAS-mCD8::GFP & + & + & + & + & + & {$[60]$} \\
\hline Gal4 [I09(2)80] UAS-mCD8::GFP & + & + & + & + & + & {$[11]$} \\
\hline NP7028 UAS-mCD8::GFP/CyO & - & - & - & + & + & [14] \\
\hline IGI-2, Gal4 [I09], UAS-GFP & + & + & + & + & + & [14] \\
\hline IGI-I UAS-GFP [S65T] UAS-mCD8::GFP & $+*$ & - & + & $?$ & $?$ & [14] \\
\hline PPkEGFP & + & + & - & + & + & {$[13]$} \\
\hline 2-2I UAS-mCD8::GFP & $+*$ & + & - & - & + & {$[16]$} \\
\hline Gr66a UAS-mCD8::GFP & $+*$ & - & - & $++\dagger$ & - & [38] \\
\hline RluAI-Gal4 UAS-mCD8::GFP & - & + & + & ++ & + & [18] \\
\hline UAS-mCD8::GFP/+; ppkGal4/+ & + & + & + & + & + & {$[61,62]$} \\
\hline NP2225 UAS-mCD8::GFP/CyO & $+*$ & - & ++ & + & + & {$[14]$} \\
\hline UAS-mCD8::GFP; CI6I UAS-mCD8::GFP/TM3 & + & + & ++ & ++ & + & [63] \\
\hline Pcol85-Gal4 UAS-mCD8::GFP/CyO Tb & - & + & - & - & + & [64] \\
\hline
\end{tabular}

Labeled neurons were identified in whole-mount adults. ++ , Strong signal; + , signals were detected with variable intensity among strains; -, signal not detected; ?, difficult to judge due to other close-by signals such as those of oenocytes. *ddaD was labeled only in A5 and A6. †Either IdaA or IdaA-like was labeled. Generally, the entire dendritic arbor of each neuron was more difficult to image just after eclosion than in 3-day or older adults because of signals from other cells such as those of the epidermis.

studied how the PCD of these two cell types is related, what the physiological significance of the spatiotemporally distinct PCD is and, more fundamentally, what the function of ddaE in pupae and young adults is. We have found strains in which ddaE in A2 to A4 persisted for at least 2 weeks with complete penetrance (KS, KK, and TU, unpublished data). Such strains would be helpful to reveal the underlying mechanisms responsible for the relatively late PCD of ddaE in A2 to A4.

\section{Pupal andlor adult da neurons provide models whereby we can study intrinsic and extrinsic mechanisms of dendritic morphogenesis and life-long maintenance}

In addition to the investigation on the control of PCD described above, pupal and/or adult da neurons provide appropriate models to address other questions regarding arbor-shaping mechanisms. In the sense of a cell-intrinsic mechanism that involves class-selective transcription factors, there were three intriguing findings in pharate adults. First, the class IV neurons (ddaC and v'ada), which had hardly expressed $\mathrm{Kn}$ at the mature larval stage, restarted expressing it when they were regenerating complex and expansive arbors. Second, ddaC and v'ada also expressed $\mathrm{Ct}$, as they do in embryonic and larval class IV neurons. Third, class I ddaE transformed its simple comb-like arbor at the larval stage into a highly bushy one without expressing the class I-selective transcription factor Ab. Whether this transcription factor-mediated control plays pivotal roles in dendritic remodeling at pupal stages or not should be tested by either knocking down $k t$ or $c t$ in the class IV neurons or misexpressing $a b$ in class I ddaE neurons selectively during the remodeling phase and then examining the effects on arbor shape. On the other hand, the radial-to-lattice remodeling of the v'ada arbor during post-emergence branch growth implies the possibility that the branch growth is guided by an extrinsic cue(s). One candidate of this cue would be fibers of the lateral tergosternal muscles, which run along the dorsal-ventral axis in the pleura of each segment and become thinner after eclosion [43]. The potential contributions of the intrinsic transcriptional control and the extrinsic cue should be examined, and it should be eventually addressed how critical arbor shape is for ddaE or v'ada to mediate cell typespecific sensory inputs.

Once the lattice arbors of v'ada neurons were generated they persisted throughout adult life, which is 50 days on average. A previous study on class IV neurons showed a pivotal role for the signaling cascade including the Ste-20related tumor suppressor kinase Hippo (Hpo) in the establishment and subsequent maintenance of dendritic fields during larval development $[44,45]$. Although dendritic arbors of adult da neurons have to persist for a much longer period than larval development (a total of 5 days), it deserves to be examined whether the larval and adult neurons share the same mechanism to maintain their dendrites or not. The mechanisms that maintain dendritic arbors have been a subject of intense investigation because defective maintenance precedes pathological conditions such as cell death in neurodegeneration [46-48]. One group of gene products responsible for neurodegeneration is associated with mitochondria, and dysfunction of mitochondria in v'ada led to the development of a sparse lattice, which degenerated progressively (A Tsub- 
ouchi, TT, and TU, submitted). Therefore, the adult da neuron would provide a novel tractable model for a systematic search for neurodegenerative mutations.

\section{Long-term in vivo time-lapse recordings of pupal da neurons}

It has been recently demonstrated in larvae that several organelles and cargos of motor proteins play critical roles in shaping dendritic arbors of da neurons, including Golgi outposts, RNA particles, and endosomes [49-53]. Unfortunately, data collection on organelle behaviors is limited in larval da neurons due to technical difficulties in keeping da neurons in mounted larvae healthy for an hour or even for 10 minutes [54]. Consequently, we have few data on the organelle behaviors that underlie the dynamics of branch elongation, sprouting, and retraction from budding of primary branches to maturation of entire dendritic arbors. One prominent advantage of observing pupal da neurons is that they are accessible to long-term in vivo time-lapse recordings. Regrowth and elaboration of the ddaE dendrite can be tracked for up to nearly 2 days ([26]; this study). Simultaneous and long-term tracking of organelles and branches in pupae, and hopefully in adults as well, would allow much more quantitative analysis to determine cause-and-effect relationships between intracellular dynamics and dendrite morphogenesis. One ultimate goal might be to compare all those data on distinct neuronal types such as IdaA and v'ada to understand neuronal type-specific dendritic morphogenesis.

\section{Conclusion}

Our study provides systematic anatomical and developmental descriptions of multidendritic neurons in the adult Drosophila abdomen. Knowledge from this study, together with future genetic and physiological studies, will help to reveal the in vivo molecular mechanisms governing dendritic remodeling as well as de novo dendrite formation, PCD of the adult neuron, and the life-long maintenance of dendritic arbors, and how each of these mechanisms is under the control of neuronal activity.

\section{Materials and methods Drosophila strains}

We used the Gal4-UAS system [55] to express most of the transgenes used and to visualize da neurons. Gal4 lines employed in this study to mark da neurons and related references are listed in Table 2. pxn-Gal4 [56] was used to visualize hemocytes in pupae. Previously reported UAS fly stocks used were UAS-GFP [S65T], UAS-mCD8::GFP, $U A S-m y r-m R F P$, and UAS-Red Stinger (all provided by the Bloomington Stock Center), yw hsflp;UAS-FRT-CD2, y FRT-mCD8::GFP/CyO; TM2/TM6B [57], and UAS-p35 [58]. We generated transgenic flies that expressed a fusion protein of mCD8 and three tandem copies of EGFP as a membrane marker (see Molecular cloning). All fly embryos, larvae, pupae, and adults were grown at $25^{\circ} \mathrm{C}$, except for in the experiment shown in Figure 6I-K, in which adults were aged at $29^{\circ} \mathrm{C}$ [59].

\section{MARCM analysis and flipout-induced GFP expression}

MARCM analyses were performed basically as described previously $[36,50]$. To label clones with mCD8::GFP, we mated either: FRT19A/FRT19A females with hsflp tubGal80 FRT19A/Y; Gal4109(2)80 UAS-mCD8::GFP/ Gal4109(2)80 UAS-mCD8::GFP males; FRT2A/FRT2A females with hsflp/Y; Gal4109(2)80 UAS-mCD8::GFP; tubGal80 FRT2A/SM5-TM6B males; or FRTG13 L Sp/SM6B males with Gal4elav[C155] UAS- $m$ CD8::GFP hsflp/ Gal4elav[C155] UAS-mCD8::GFP hsflp; tub-Gal80 FRTG13/ tub-Gal80 FRTG13 females. For flipout-induced cell labeling with GFP, $y w$ hsflp;UAS-FRT-CD2, $y^{+-F R T-}$ $m C D 8:: G F P / C y O$; TM2/TM6B males were mated with C161/TMGB females; and the recovered embryos and early larvae were treated with a heat shock $\left(37^{\circ} \mathrm{C}\right.$ for 30 minutes). Adults with GFP-expressing clones were dissected, fixed, and stained for markers. Detailed genotypes of the animals and clones are described in the legends of individual figures.

\section{Immunohistochemistry}

White prepupae were collected 0 to $0.5 \mathrm{~h} \mathrm{APF}$ and incubated at $25^{\circ} \mathrm{C}$. At appropriate ages, each pupa was placed on double-sticky scotch tape, taken out of its pupal case (puparium), and its thorax pinned down. We covered the pupa with a drop of phosphate-buffered saline, nicked the posterior end of the abdomen, and then cut it in an anterior direction. Adult flies were dissected in a similar way. The opened abdomen was pinned down when necessary, washed in phosphate-buffered saline, and subsequently fixed in phosphate-buffered saline containing $0.1 \%$ Triton X-100 (PBT) and 3.7\% formaldehyde at room temperature for 30 minutes. After having been washed with PBT, these fillet preparations were stained according to standard protocols with the following primary antibodies: rabbit anti-GFP (Molecular Probes, Eugene, OR, USA), rat anti-mCD8 alpha subunit (Caltag, Burlingname, Californina), rabbit anti-HRP (Jackson ImmunoResearch_West Grove, PA, USA), mouse antiElav (Developmental Studies Hybridoma Bank, DSHB), rat anti-Kn [17], rabbit anti-Ab [21], mouse anti-Ct (DSHB), mouse anti-CD2 (Serotec_Oxford, UK), and mouse 22C10 (DSHB). Thoraxes were removed either just after fixation or before mounting the preparations. The abdomens were mounted in FluorSave (Calbiochem, Darmstadt, Germany) and viewed with a laser scanning confocal microscope (Zeiss LSM 510 META, Nikon C1, Leica TCS SPE, or BioRad MRC1024) with a 0.9 or $1.5 \mu \mathrm{m}$ Z-step. Preservation of peripheral axonal fascicles in the abdomen was sometimes difficult, but improved when a posterior part of the ventral ganglion was left instead of 
removing the entire thorax. Images of serial optical sections were overlaid to generate each panel in the figures.

\section{Image acquisition of whole-mount animals and quantitative analysis}

Imaging da neurons in whole-mount larvae was done as described earlier $[17,50]$. For imaging pupal da neurons, each pupa that had been taken out of its puparium was put on a $35-\mathrm{mm}$ glass-bottomed dish (IWAKI, Tokyo, Japan). To prevent desiccation during our time-lapse recordings, we placed a wet filter paper in the dish. After image acquisition, typically with 5\% laser power, we kept every pupa at $25^{\circ} \mathrm{C}$ and confirmed that they survived to the adult stage. Segments observed were identified on the basis of landmarks such as spiracles and larval persisting muscles [43]. To acquire images of da neurons in adults, we removed the heads, wings, and legs of adult flies and mounted the abdomens in 50\% glycerol on slides in between spacers made of vinyl tape. All of the images were acquired using laser scanning confocal microscopy. For quantification of dendritic patterns of da neurons, we used Neurocyte software (Kurabo, Osaka, Japan).

\section{Molecular cloning}

We constructed two plasmids, pCaspeR-ppk-hs43mCD8::3xEGFP and pUAST [mCD8::3xEGFP], and generated trasngenic flies. Briefly, a cDNA fragment encoding mCD8 was amplified from genomic DNA of the pUAST [mCD8::GFP] stock (the Bloomington Stock Center) and ligated with a plasmid that had the open reading frame of 3xEGFP (T Harumoto and TU, unpublished). The fragment encoding mCD8::3xEGFP was recovered and ligated with fragments derived from pCaspeR-ppk-hs43-EGFP ([13]) and pUAST [55] to construct pCaspeR-ppk-hs43mCD8::3xEGFP and pUAST [mCD8::3xEGFP], respectively.

\section{Abbreviations}

Ab: Abrupt; APF: after puparium formation; Ct: Cut; da: dendritic arborization; dbd: dorsal bipolar dendrite; DIOM: dorsal internal oblique muscle; DSHB: Developmental Studies Hybridoma Bank; es: external sensory; EGFP: enhanced GFP; GFP: green fluorescent protein; HRP: horse radish peroxidase; Kn: Knot; PCD: programmed cell death.

\section{Competing interests}

The authors declare that they have no competing interests.

\section{Authors' contributions}

$\mathrm{KS}, \mathrm{AF}, \mathrm{MS}$, and KK carried out all immunohistochemical and imaging experiments whose results are shown in the figures. TT, YH, KS, and MY-K performed those experiments at the initial stages of this research. T Us helped with molecular cloning, and T Ue wrote the manuscript.

\section{Additional material}

\section{Additional file 1}

Dendritic pruning and migrating hemocytes. Class IV ddaC and hemocytes were imaged at 5-minute intervals with a $1.5 \mu \mathrm{m}$ Z-step between 4 h 50 minutes APF and 6 h APF. Dendritic branches became detached from the cell body and eventually disappeared. The hemocytes were migrating by generating prominent lamellipodia. Genotype: UAS-EGFP; ppk-EGFP/pxn-Gal4.

Click here for file

[http://www.biomedcentral.com/content/supplementary/1749-

8104-4-37-S1.MOV]

\section{Additional file 2}

Identification of da neurons in live whole-mount pupae. (A) Timelapse recordings of A4 tergite of a whole-mount pupa of genotype Gal4 ${ }^{109(2) 80}$ UAS-mCD8::GFP/Gal4109(2)80 UAS-mCD8::GFP. By 27 $h A P F$, dendrites had degenerated, and four cells (arrows at $27 \mathrm{~h} \mathrm{APF)}$ were aligned along the dorsal-ventral axis. Those four cells were identified as indicated. Scale bar: $50 \mu \mathrm{m}$. (B-H) We also observed whole-mount pupae of the subset markers and addressed whether the specificity of the marker expression persisted in pupae or not. (B-E) The class IV marker ppk-EGFP [13] primarily labeled ddaC in the tergite and v'ada in the pleura (not shown) as it did in larvae. Tergite of a whole-mount $60 \mathrm{~h} \mathrm{APF}$ pupa of genotype Gal4109(2)80 UAS-mmRFP/Gal4109(2)80 UASmmRFP; ppk-GFP/ppk-EGFP. The channel signal of RFP (B), GFP $(C)$, the merge of these $(D)$, and tracings $(E)$ are shown. $(F-H)$ Example of other markers for 'subsets' of larval da neuons. A3 or A4 tergites of whole-mount 60 h APF pupae of genotype ppk-Gal4 UAS-mCD8::GFP/ ppk-Gal4 UAS-mCD8::GFP (F), which labeled all da neurons, Gal 4477 UAS-mCD8::GFP/Gal4477 UAS-mCD8::GFP (G), which labeled both d $a$ and es neurons, and UAS-mCD8::GFP/UAS-mCD8::GFP; C161/ TM3 (H). In (H), ddaC was weakly labeled and difficult to see in this sample. Expression profiles of various Gal4 lines in the adult are summarized in Table 2. Scale bars: $50 \mu \mathrm{m}$.

Click here for file

[http://www.biomedcentral.com/content/supplementary/1749-

8104-4-37-S2.PDF]

\section{Additional file 3}

ddaD and ldaA/ldaA-like in adults. (A) Tracings of dendritic arbors of ddaD clones in A3/A4 (top) and A5/A6 (bottom). Each dendritic branch of ddaD was sometimes difficult to resolve. (B) Gr66aGal4 visualizes dendritic arbors of ldaA or ldaA-like at single-cell resolution. Pleura of A2 to A6 of UAS-RedStinger/+; Gr66aGal4 UAS-mCD8::GFP/+ was imaged through the channel of GFP (green) and DsRed (magenta; overlap with green, white). In each hemisegment, the nucleus of a single neuron (ldaA or lda-like) was labeled (inset; compare with that in Figure 5M). Arrows point to cell bodies. sp: spiracle. Scale bars: $50 \mu \mathrm{m}$ (A); 100 $\mu \mathrm{m}(\mathrm{B})$.

Click here for file

[http://www.biomedcentral.com/content/supplementary/17498104-4-37-S3.PDF] 


\section{Additional file 4}

Segment-dependent variability in the composition of da neurons in the tergite. (A) A fillet preparation of a pharate adult female was stained with anti-HRP antibody. This image is a high-power view of the boxed region in Figure 2E. Yellow brackets indicate dendritic arbors of ddaE in A2 to A4; ddaE-like cells that formed such bushy dendritic arbors were not found in A5 or A6 $(n=9)$. Yellow arrowheads indicate cell bodies of representative es neurons, and blue arrows dendrites of dbds. Genotype: Gal4109(2)80 UAS-mCD8::GFP/Gal4109(2)80 UAS-mCD8::GFP. Scale bar: $50 \mu \mathrm{m}$. (B-F) Another fillet preparation of the same genotype as in (A), which was doubly stained with anti-mCD8 antibody $(B, C-F)$ and antibody against a pan-neuronal nuclear protein, Elav $(C-F)$. Boxed regions of $A 2$ to $A 5$ in $(B)$ are highlighted in (C-F). (C-F) Three panels of each hemisegment show $m C D 8$ staining (left; green in the right panel), Elav staining (middle; magenta or white in the right panel), and a merged image (right panel). Yellow arrows point to nuclei of da neurons, and blue arrows to those of $d b d$. Other smaller Elav-positive nuclei are those of es neurons. Typically, the number of the neuronal nuclei of da in the tergite was two in $A 5$ and $A 6(F)$, in comparison to three in $A 3$ and $A 4(D, E)$. Together with the results shown in $(A)$ and Figure $8 A$, these results strongly suggest that ddaE was absent in $A 5$ and $A 6$ in the pharate adult. The A2 tergite had two, not three, da neurons (C). Our observation using ppk-EGFP (a marker of ddaC; Additional file 2CC) and C161 Gal4 (a marker of ddaE; Figure 8A) showed that A2 had ddaC and ddaE, but not ddaD. (G) Collectively, the composition of da neurons in each segment at the pharate stage is summarized. Plus and minus signs represent the presence and absence of each neuronal type, respectively, and a plus sign with an asterisk means cell death within 1 week after eclosion. Scale bars: $100 \mu \mathrm{m}$ (B); $50 \mu \mathrm{m}$ (C-F).

Click here for file

[http://www.biomedcentral.com/content/supplementary/1749-

8104-4-37-S4.PDF]

\section{Additional file 5}

Degeneration of ddaE in A5. Time-lapse recording of tergites of A4 and A5 (right). Images were taken every 10 minutes with a $2 \mu \mathrm{m}$ Z-step between $14 \mathrm{~h} A P F$ and $62 \mathrm{~h}$ APF. A branch of ddaE in A5 was degraded after it started regeneration. Towards the end of the movie, the dorsal internal oblique muscle 1 (DIOM1) is seen at the top (dorsal) in both A4 and $\mathrm{A} 5$, whereas DIOM3 was present at a lateral position in only $\mathrm{A} 4$. We performed time-lapse recordings of pupae of this genotype a total of four times. Genotype: UAS-mCD8::GFP/UAS-mCD8::GFP; C161 UASmCD8::GFP/TM3 Ser Sb.

Click here for file

[http://www.biomedcentral.com/content/supplementary/17498104-4-37-S5.MOV]

\section{Acknowledgements}

The Developmental Studies Hybridoma Bank at the University of lowa, the Bloomington Stock Center, and the Drosophila Genetic Resource Center at Kyoto Institute of Technology provided the antibodies and fly stocks. We thank K Emoto, $\mathrm{K}$ Yasunaga, and $\mathrm{YN}$ Jan for communicating data prior to publication. We are also grateful to YN Jan, B Yi, Y Zhang, S Sheperd, CL Hughes, A Moore, D Williams, M A Crozatier, and T Tabata for providing other fly strains and reagents. We thank very much $T$ Tanimura, $T$ Inoshita, A Tsubouchi, and D Satoh for their critical advice and encouragement; and Y Miyake, K Shimizu, T Nomura, and M Futamata for their technical assistance. This work was supported by grants from the programs Grants-in-Aid for Scientific Research on Priority Areas-Molecular Brain Science (17024025 to TU) of the MEXT of Japan.

\section{References}

I. Truman JW: Developmental neuroethology of insect metamorphosis. J Neurobiol 1992, 23: | 404- I422.

2. Consoulas C, Duch C, Bayline RJ, Levine RB: Behavioral transformations during metamorphosis: remodeling of neural and motor systems. Brain Res Bull 2000, 53:57I-583.

3. Wong RO, Ghosh A: Activity-dependent regulation of dendritic growth and patterning. Nat Rev Neurosci 2002, 3:803-8I2.

4. Luo L, O'Leary DD: Axon retraction and degeneration in development and disease. Annu Rev Neurosci 2005, 28: I 27-I 56.

5. Consoulas C, Restifo LL, Levine RB: Dendritic remodeling and growth of motoneurons during metamorphosis of Drosophila melanogaster. J Neurosci 2002, 22:4906-49I7.

6. Tissot M, Stocker RF: Metamorphosis in drosophila and other insects: the fate of neurons throughout the stages. Prog Neurobiol 2000, 62:89-III.

7. Levine RB, Truman JW: Metamorphosis of the insect nervous system: changes in morphology and synaptic interactions of identified neurones. Nature 1982, 299:250-252.

8. Corty MM, Matthews BJ, Grueber WB: Molecules and mechanisms of dendrite development in Drosophila. Development 2009, I36: 1049-1061.

9. Parrish JZ, Emoto K, Kim MD, Jan YN: Mechanisms that regulate establishment, maintenance, and remodeling of dendritic fields. Annu Rev Neurosci 2007, 30:399-423.

10. Bodmer R, Barbel S, Sheperd S, Jack JW, Jan LY, Jan YN: Transformation of sensory organs by mutations of the cut locus of $D$. melanogaster. Cell 1987, 5 I:293-307.

II. Gao FB, Brenman JE, Jan LY, Jan YN: Genes regulating dendritic outgrowth, branching, and routing in Drosophila. Genes Dev 1999, I 3:2549-256I.

12. Grueber WB, Jan LY, Jan YN: Tiling of the Drosophila epidermis by multidendritic sensory neurons. Development 2002, I 29:2867-2878.

13. Grueber WB, Ye B, Moore AW, Jan LY, Jan YN: Dendrites of distinct classes of Drosophila sensory neurons show different capacities for homotypic repulsion. Curr Biol 2003, 13:6 I 8-626.

14. Sugimura K, Yamamoto M, Niwa R, Satoh D, Goto S, Taniguchi M, Hayashi S, Uemura T: Distinct developmental modes and lesion-induced reactions of dendrites of two classes of Drosophila sensory neurons. J Neurosci 2003, 23:3752-3760.

15. Crozatier $M$, Vincent A: Control of multidendritic neuron differentiation in Drosophila: the role of Collier. Dev Biol 2008, 3 I 5:232-242.

16. Grueber WB, Jan LY, Jan YN: Different levels of the homeodomain protein cut regulate distinct dendrite branching patterns of Drosophila multidendritic neurons. Cell 2003, I | 2:805-8|8.

17. Hattori Y, Sugimura K, Uemura T: Selective expression of Knot/ Collier, a transcriptional regulator of the EBF/OIf-I family, endows the Drosophila sensory system with neuronal classspecific elaborated dendritic patterns. Genes Cells 2007, I2: 101 1-1022.

18. Jinushi-Nakao S, Arvind R, Amikura R, Kinameri E, Liu AW, Moore AW: Knot/Collier and cut control different aspects of dendrite cytoskeleton and synergize to define final arbor shape. Neuron 2007, 56:963-978.

19. Kim MD, Jan LY, Jan YN: The bHLH-PAS protein Spineless is necessary for the diversification of dendrite morphology of Drosophila dendritic arborization neurons. Genes Dev 2006, 20:2806-2819.

20. Li W, Wang F, Menut L, Gao FB: BTB/POZ-zinc finger protein abrupt suppresses dendritic branching in a neuronal subtype-specific and dosage-dependent manner. Neuron 2004, 43:823-834

21. Sugimura K, Satoh D, Estes P, Crews S, Uemura T: Development of morphological diversity of dendrites in Drosophila by the BTB-zinc finger protein abrupt. Neuron 2004, 43:809-822.

22. Hughes CL, Thomas JB: A sensory feedback circuit coordinates muscle activity in Drosophila. Mol Cell Neurosci 2007, 35:383-396.

23. Hwang RY, Zhong L, Xu Y, Johnson T, Zhang F, Deisseroth K, Tracey WD: Nociceptive neurons protect Drosophila larvae from parasitoid wasps. Curr Biol 2007, I 7:2105-2116.

24. Song W, Onishi M, Jan LY, Jan YN: Peripheral multidendritic sensory neurons are necessary for rhythmic locomotion behav- 
ior in Drosophila larvae. Proc Natl Acad Sci USA 2007, 104:5199-5204.

25. Williams DW, Shepherd D: Persistent larval sensory neurons in adult Drosophila melanogaster. I Neurobiol 1999, 39:275-286.

26. Williams DW, Truman JW: Mechanisms of dendritic elaboration of sensory neurons in Drosophila: insights from in vivo time lapse. J Neurosci 2004, 24: I54I-I550.

27. Williams DW, Truman JW: Cellular mechanisms of dendrite pruning in Drosophila: insights from in vivo time-lapse of remodeling dendritic arborizing sensory neurons. Development 2005, 132:363|-3642.

28. Williams DW, Truman JW: Remodeling dendrites during insect metamorphosis. J Neurobiol 2005, 64:24-33.

29. Kuo CT, Jan LY, Jan YN: Dendrite-specific remodeling of Drosophila sensory neurons requires matrix metalloproteases, ubiquitin-proteasome, and ecdysone signaling. Proc Natl Acad Sci USA 2005, 102: I5230-15235.

30. Kuo CT, Zhu S, Younger S, Jan LY, Jan YN: Identification of E2/E3 ubiquitinating enzymes and caspase activity regulating Drosophila sensory neuron dendrite pruning. Neuron 2006, I I:283-290.

31. Lee HH, Jan LY, Jan YN: Drosophila IKK-related kinase Ik2 and Katanin p60-like I regulate dendrite pruning of sensory neuron during metamorphosis. Proc Natl Acad Sci USA 2009, 106:6363-8.

32. Williams DW, Kondo S, Krzyzanowska A, Hiromi Y, Truman JW: Local caspase activity directs engulfment of dendrites during pruning. Nat Neurosci 2006, 9: I 234-I 236

33. Jan LY, Jan YN: Antibodies to horseradish peroxidase as specific neuronal markers in Drosophila and in grasshopper embryos. Proc Natl Acad Sci USA 1982, 79:2700-2704.

34. Dulcis D, Levine RB: Innervation of the heart of the adult fruit fly, Drosophila melanogaster. J Comp Neurol 2003, 465:560-578.

35. Fujita SC, Zipursky SL, Benzer S, Ferrus A, Shotwell SL: Monoclona antibodies against the Drosophila nervous system. Proc Natl Acad Sci USA 1982, 79:7929-7933.

36. Lee T, Luo L: Mosaic analysis with a repressible cell marker for studies of gene function in neuronal morphogenesis. Neuron 1999, 22:45|-46|.

37. Yang CH, Rumpf S, Xiang Y, Gordon MD, Song W, Jan LY, Jan YN Control of the postmating behavioral switch in Drosophila females by internal sensory neurons. Neuron 2009, 61:519-526.

38. Dunipace L, Meister S, McNealy C, Amrein H: Spatially restricted expression of candidate taste receptors in the Drosophila gustatory system. Curr Biol 200 I, I I:822-835.

39. Blagburn JM: Engrailed expression in subsets of adult Drosophila sensory neurons: an enhancer-trap study. Invert Neurosci 2008, 8: |33-|46.

40. Lee Y, Moon SJ, Montell C: Multiple gustatory receptors required for the caffeine response in Drosophila. Proc Natl Acad Sci USA 2009, 106:4495-4500.

4I. Moon SJ, Kottgen M, Jiao Y, Xu H, Montell C: A taste receptor required for the caffeine response in vivo. Curr Biol 2006, 16:1812-1817.

42. Hiroi M, Tanimura T, Marion-Poll F: Hedonic taste in Drosophila revealed by olfactory receptors expressed in taste neurons. PLoS ONE 2008, 3:e2610.

43. Kimura KI, Truman JW: Postmetamorphic cell death in the nervous and muscular systems of Drosophila melanogaster. | Neurosci 1990, 10:403-40|

44. Emoto K, Parrish JZ, Jan LY, Jan YN: The tumour suppressor Hippo acts with the NDR kinases in dendritic tiling and maintenance. Nature 2006, 443:210-213.

45. Parrish JZ, Emoto K, Jan LY, Jan YN: Polycomb genes interact with the tumor suppressor genes hippo and warts in the maintenance of Drosophila sensory neuron dendrites. Genes Dev 2007, $21: 956-972$

46. $\mathrm{Li} \mathrm{H}, \mathrm{Li} \mathrm{SH}, \mathrm{Yu} Z \mathrm{ZX}$, Shelbourne P, Li XJ: Huntingtin aggregateassociated axonal degeneration is an early pathologica event in Huntington's disease mice. I Neurosci 200I, 21:8473-848I.

47. Raff MC, Whitmore AV, Finn JT: Axonal self-destruction and neurodegeneration. Science 2002, 296:868-87I.

48. Tsai J, Grutzendler J, Duff K, Gan WB: Fibrillar amyloid deposition leads to local synaptic abnormalities and breakage of neuronal branches. Nat Neurosci 2004, 7: | I8I-I I83.
49. Brechbiel JL, Gavis ER: Spatial regulation of nanos is required for its function in dendrite morphogenesis. Curr Biol 2008, 18:745-750.

50. Satoh D, Sato D, Tsuyama T, Saito M, Ohkura H, Rolls MM, Ishikawa F, Uemura T: Spatial control of branching within dendritic arbors by dynein-dependent transport of Rab5-endosomes. Nat Cell Biol 2008, I 0: I 164-I I7I.

51. Sweeney NT, Brenman JE, Jan YN, Gao FB: The coiled-coil protein shrub controls neuronal morphogenesis in Drosophila. Curr Biol 2006, 16:1006-1011.

52. Ye B, Zhang Y, Song W, Younger SH, Jan LY, Jan YN: Growing dendrites and axons differ in their reliance on the secretory pathway. Cell 2007, I30:717-729.

53. Zheng $Y$, Wildonger J, Ye B, Zhang $Y$, Kita A, Younger SH, Zimmerman $\mathrm{S}$, Jan $\mathrm{LY}$, Jan $Y N$ : Dynein is required for polarized dendritic transport and uniform microtubule orientation in axons. Nat Cell Biol 2008, 10:1 I72-1 I80.

54. Rolls MM, Satoh D, Clyne PJ, Henner AL, Uemura T, Doe CQ: Polarity and intracellular compartmentalization of Drosophila neurons. Neural Dev 2007, 2:7.

55. Brand $\mathrm{AH}$, Perrimon $\mathrm{N}$ : Targeted gene expression as a means of altering cell fates and generating dominant phenotypes. Development 1993, II 8:40|-4I5

56. Stramer B, Wood W, Galko MJ, Redd MJ, Jacinto A, Parkhurst SM, Martin P: Live imaging of wound inflammation in Drosophila embryos reveals key roles for small GTPases during in vivo cell migration. J Cell Biol 2005, 168:567-573.

57. Wang Z, Singhvi A, Kong P, Scott K: Taste representations in the Drosophila brain. Cell 2004, I I 7:98I-99I.

58. Yoo SJ, Huh JR, Muro I, Yu H, Wang L, Wang SL, Feldman RM, Clem RJ, Muller HA, Hay BA: Hid, Rpr and Grim negatively regulate DIAPI levels through distinct mechanisms. Nat Cell Biol 2002, 4:416-424.

59. Min KT, Benzer S: Spongecake and eggroll: two hereditary diseases in Drosophila resemble patterns of human brain degeneration. Curr Biol 1997, 7:885-888.

60. Lin DM, Goodman CS: Ectopic and increased expression of Fasciclin II alters motoneuron growth cone guidance. Neuron 1994, 13:507-523.

6I. Kimura H, Usui T, Tsubouchi A, Uemura T: Potential dual molecular interaction of the Drosophila 7-pass transmembrane cadherin Flamingo in dendritic morphogenesis. J Cell Sci 2006, I 19:1 I 18-1129.

62. Ainsley JA, Pettus JM, Bosenko D, Gerstein CE, Zinkevich N, Anderson MG, Adams CM, Welsh MJ, Johnson WA: Enhanced locomotion caused by loss of the Drosophila DEG/ENaC protein Pickpocket I. Curr Biol 2003, 13:1557-1563.

63. Shepherd D, Smith SA: Central projections of persistent larval sensory neurons prefigure adult sensory pathways in the CNS of Drosophila. Development 1996, 122:2375-2384.

64. Krzemien J, Dubois L, Makki R, Meister M, Vincent A, Crozatier M: Control of blood cell homeostasis in Drosophila larvae by the posterior signalling centre. Nature 2007, 446:325-328.

Publish with Bio Med Central and every scientist can read your work free of charge

"BioMed Central will be the most significant development for disseminating the results of biomedical research in our lifetime. "

Sir Paul Nurse, Cancer Research UK

Your research papers will be:

- available free of charge to the entire biomedical community

- peer reviewed and published immediately upon acceptance

- cited in PubMed and archived on PubMed Central

- yours - you keep the copyright 